Cite this: Energy Environ. Sci., 2014, 7, 2145

\title{
Morphology control strategies for solution- processed organic semiconductor thin films
}

\author{
Ying Diao, ${ }^{\text {ab }}$ Leo Shaw, $^{a}$ Zhenan Bao*a and Stefan C. B. Mannsfeld ${ }^{\star b}$
}

While the chemical structure of organic semiconductors has an obvious effect on their proclivity for charge transport, the ways with which they are processed have a dramatic effect on the performance of plastic electronics devices incorporating them. In some cases, morphological defects and misalignment of crystalline grains can completely obscure the materials' intrinsic charge transport properties. Although some deposition methods, especially vapor-phase ones, can produce single crystals and thus avoid some of these problems, it is desirable to gain a fundamental understanding of how to improve charge transport when using solution-phase deposition techniques. In this review, we present both a survey of solution-based processing techniques for plastic electronics relevant on both the commercial and research scale and a set of strategies to control thin film morphology towards enhancing their electronic transport properties.

Received 28th February 2014

Accepted 7th May 2014

DOI: $10.1039 / \mathrm{c} 4 \mathrm{ee} 00688 \mathrm{~g}$

www.rsc.org/ees properties.

\begin{abstract}
Broader context
Conventional inorganic semiconductor materials and devices are currently manufactured using a top-down fabrication approach involving multiple steps of high temperature processing at thousands of Fahrenheit. In comparison, organic semiconductors can be made using more energy-efficient and cost-effective methods at near ambient conditions, such as roll-to-roll solution printing - a bottom-up processing method by which newspapers are manufactured. Such a solution also enables large-area deposition on plastic substrates and therefore flexible electronic devices. One of the major challenges to achieving solutionprocessed organic semiconductors is the control of thin film morphology during printing/coating processes, which critically influences the device performance, often by orders of magnitude. With the recent invention of numerous solution-processing methods emerged many elegant approaches for controlling thin-film morphology, specifically, the control of nucleation, crystal growth, in-plane and out-of-plane domain alignment, etc. In this review, we highlight these recent advancements in morphology control strategies in the context of solution-processed organic semiconductors, and their impact on the electronic properties of the resulting devices. It can be expected that the understanding and control of thin-film morphology during solution processing will bring us closer to the future of energy-efficient production of low-cost, high-performance flexible electronic devices.
\end{abstract}

\section{Introduction}

Although many of the pioneering studies in the physics of organic semiconductors (OSCs) relied on vapor-grown crystals, there has been a significant interest recently in solution-based deposition techniques. While some of the highest charge carrier mobilities in OSCs have been observed in single crystals obtained by vapor-phase deposition, the performance of devices incorporating solution-grown OSC thin films and single crystals have improved dramatically in the past few years. Even though new synthetic routes and design principles have been used to create novel semiconductor molecules, the improvements to device performance largely follow from the elucidation of the role of morphology and alignment in the charge transport efficiency of thin films. Indeed, such knowledge has cast a light on the importance of a comprehensive

a Department of Chemical Engineering, Stanford University, Stanford, California 94305, USA. E-mail: zbao@stanford.edu

${ }^{b}$ Stanford Synchrotron Radiation Lightsource, SLAC National Accelerator Laboratory, Menlo Park, California 94025, USA. E-mail: mannsfel@slac.stanford.edu understanding of the physical principles underlying various processing mechanisms. An appreciation for fundamental studies along these lines can give us insight into how to control various aspects of these solution deposition techniques to achieve the desired performance of organic electronic devices.

The goal of this review is twofold: (1) to survey and introduce a variety of solution-based deposition techniques commonly used in the industry and in research on organic electronics, and (2) to identify strategies used to tune the alignment and morphology of OSC thin films. It is our hope that the methods described here can inspire both the application of these methodologies to other processing techniques and the development of new approaches for controlling these properties.

\section{Solution-based processing techniques}

In this section, we will introduce and describe an array of processing methods that use organic semiconductor solutions. The 
variety and diversity of techniques attests to the versatility of solution-phase deposition, and we attempt to give a representative sampling of exemplary methods discussed in the literature (Fig. 1).

\subsection{Dropcasting}

A facile quasi-equilibrium process, dropcasting involves the casting of an OSC solution and the subsequent evaporation of the solvent to precipitate and deposit either individual crystals or a thin film. For OSCs with strong self-organizing behavior, single crystals can be obtained directly from dropcasting or from another recrystallization process. In the latter, a multistep process would consist of suspending the crystals in a nonsolvent and casting them again on a target substrate. One-step processes are also possible and would comprise the direct formation of crystals or thin films by one dropcasting event. A variety of modifications have been developed to enhance the quality of deposited crystals and thin films. For example, vibration-assisted crystallization - where the dropcast solution is exposed to unidirectional sound waves $(\sim 100 \mathrm{~Hz})$ during evaporation - was found to enhance crystal quality and device performance. ${ }^{1}$ In other cases, control of solvent evaporation was achieved by using mixed solvents, azeotropic mixtures, ${ }^{2}$ sealed chambers, ${ }^{3,4}$ saturated solvent environments, ${ }^{5}$ inert gas purging, ${ }^{6}$ and surface treatments. ${ }^{7}$

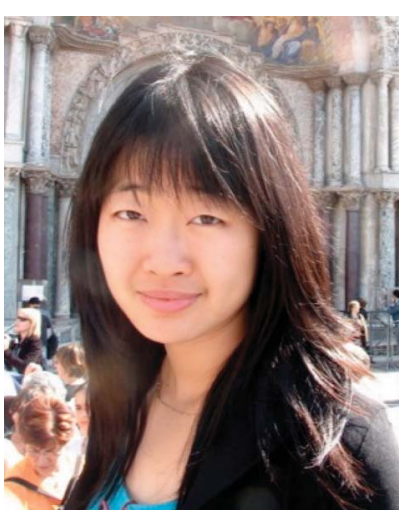

Ying Diao earned her B.S. degree from Tsinghua University before she joined MIT in September, 2011 to pursue graduate education in Chemical Engineering, working on designing polymeric surfaces for controlling crystallization of pharmaceutical compounds and on understanding the nanoconfinement effect on nucleation from solution. She is currently a postdoctoral scholar working with Prof. Zhenan Bao in the Department of Chemical Engineering at Stanford, Dr. Stefan Mannsfeld, and Dr. Michael Toney at the SLAC National Accelerator Laboratory. Her research topics include solution printing of organic semiconductor thin films and in-situ structural characterization using $X$-ray scattering techniques. She will join University of Illinois, Urbana-Champaign in 2015 as an assistant professor in the Department of Chemical Engineering to pursue her interest in controlled molecular assembly at interfaces for energy and biomedical applications.

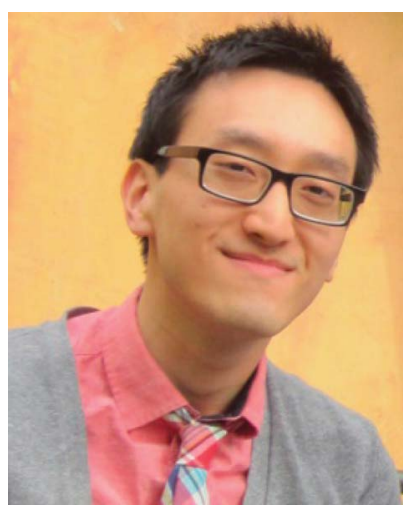

Leo Shaw was born and raised in warm and sunny southern California. For his undergraduate thesis, he worked on the synthesis and characterization of small molecular organic semiconductors under the guidance of Professor Yueh-Lin (Lynn) Loo. He graduated with a B.S.E. degree in chemical and biological engineering from Princeton University in 2012. At Stanford University, he is pursuing a M.S. in electrical engineering and is a Ph.D. candidate in chemical engineering. He became a member of Professor Zhenan Bao's research group in 2013 and began work on the development of solution shearing as a tool for high quality organic semiconductor single crystal deposition.

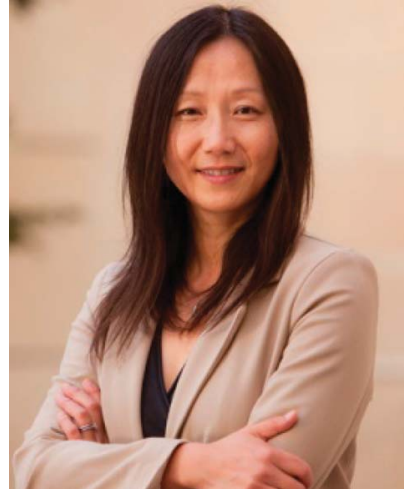

Zhenan Bao is a Professor of Chemical Engineering at Stanford University, and by courtesy a Professor of Chemistry and Material Science and Engineering. Prior to joining Stanford in 2004, she was a Distinguished Member of Technical Staff in Bell Labs, Lucent Technologies from 1995-2004. She has over 300 refereed publications and over 40 US patents. Bao is a Fellow of ACS, AAAS, SPIE, ACS PMSE and ACS POLY. She served as a Board Member for the National Academy Board on Chemical Sciences and Technology and Board of Directors for the Materials Research Society. She is a recipient of the ACS Polymer Division Carl S. Marvel Creative Polymer Chemistry Award 2013, ACS Author Cope Scholar Award 2011, Royal Society of Chemistry Beilby Medal and Prize 2009, IUPAC Creativity in Applied Polymer Science Prize 2008, ACS Team Innovation Award 2001, and the R\&D 100 Award 2001.

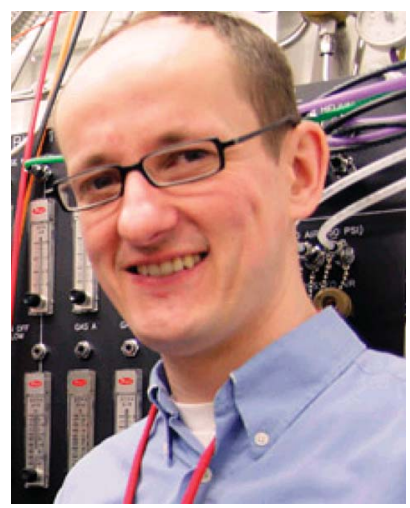

Stefan Mannsfeld joined the Materials Science Department of the Stanford Synchrotron Radiation Lightsource as a Staff Scientist in 2009. He obtained his Ph.D. in 2004 from the Dresden University of Technology (Germany) after which he was a postdoctoral scholar at the Department of Chemical Engineering, Stanford University. He coauthored more than 65 peer-reviewed journal publications and received the 2011 William E. and Diane M. Spicer Young Investigator Award for his research in Organic Electronics. In Fall 2014, he will join the Center for Advancing Electronics Dresden (cfAED) at the Dresden University of Technology as Professor for Organic Electronic Devices. 
2.1.1 Droplet pinning. A major category of modified dropcasting methods is droplet pinning. A solid structure, often a piece of silicon or plastic, is used to pin a solution droplet so there is steady contraction of the three-phase contact line. In the case of dropcasting without a pinner, the solution droplet is free to move about the substrate, and the recession of the contact line is irregular, leading to unsteady, nonuniform deposition. ${ }^{8}$ At the beginning of solvent evaporation from the droplet, nucleation events occur all along the edge of the droplet and effectively guide the subsequent growth of (single-crystalline) ribbons and needles aligned in the direction of contact line recession. ${ }^{\mathbf{9 , 1 0}}$ Because patterning is essentially dictated by the position of pinners, large-area deposition of aligned OSC crystals has been demonstrated using multiple pinners. There are also a few implementations of droplet pinning that involve the use of inclined substrates to induce alignment. ${ }^{11-13}$ So far, the pinning of inclined droplets have allowed for thin films to be deposited, in contrast to the multiple crystals formed from droplet pinning on flat substrates.

\subsection{Spincoating}

Spincoating is a commonly used solution method to form OSC thin films of effectively uniform thickness. The solution is dropped onto a substrate, and the substrate is accelerated to a high angular velocity to simultaneously spread the liquid and evaporate the solvent. The thickness of the wet film is inversely related to the spin speed and also depends on the solution concentration and viscosity. Spincoating is most often used to deposit the active layer, but there has also been research looking into the simultaneous, one-step deposition of both the semiconductor and the dielectric layer needed for many device applications. ${ }^{14}$ The vertical phase segregation and self-assembly of OSC/insulator pairs led to improvements in morphology and device performance in several instances. ${ }^{15,16}$
Modifications of conventional spincoating have been used to fabricate high performance devices. Yuan et al. used an "offcenter" method whereby the target substrate was placed 20 to 40 $\mathrm{mm}$ away from the central rotation axis. ${ }^{17}$ The centrifugal force in this modified technique - unlike the case of on-center spincoating, where solution is spread radially outward - facilitated unidirectional alignment of the resulting OSC thin film. Because of the kinetic nature of the process and the rapid solvent evaporation and film formation, a high performance, metastable molecular packing was achieved.

\subsection{Meniscus-guided coating}

Several solution coating techniques use the linear translation of either the substrate or the coating tool to induce aligned crystallite growth in the deposited thin films. These methods involve the evolution of a solution meniscus, which acts as an air-liquid interface for solvent evaporation. The solution concentrates with the removal of solvent, and once the point of supersaturation is reached, the solute precipitates and is deposited as thin film. In many of these techniques, alignment of the growing OSC thin film is achieved by virtue of the inherent directionality of the linear motion guiding the solution.

2.3.1 Dipcoating. Much like how it sounds, dipcoating involves the vertical withdrawal of a substrate dipped in a bath of OSC solution. Here, key parameters such as withdrawal velocity and substrate/solution temperature influence the development of concentration gradients and fluid flow within the meniscus. Depending on the solvent evaporation rate and the substrate speed, wet films of varying thicknesses are achievable and can produce aligned crystalline domains in the dried films. The free (liquid-air) and fixed (liquid-solid) interfaces are also relevant boundary conditions when considering the fluid mechanics of these systems. Solvent choice is especially important because of its effect on the rate of solvent

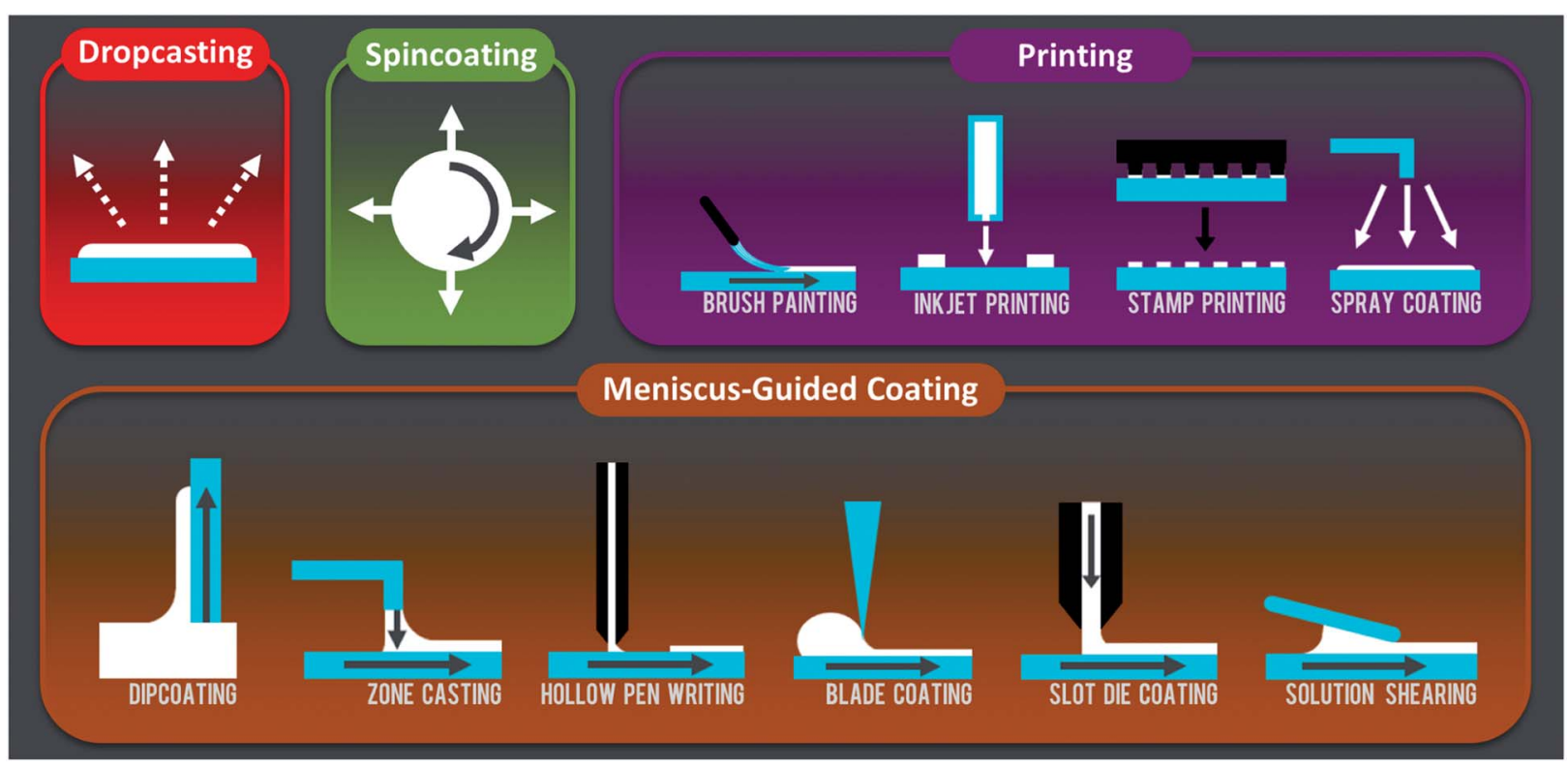

Fig. 1 A schematic summary of the solution-based deposition techniques discussed. 
evaporation. Binary azeotropic solvent mixtures, for example, have been exploited, yielding improvements to both the film morphology and the performance of devices incorporating deposited OSC thin films. ${ }^{18}$

2.3.2 Zone casting. The name of this technique is derived from the familiar zone refining (or floating zone) process, which is commonly used to create high purity inorganic semiconductor single crystals. Zone refining was also shown to be possible for organic materials using a heating wire to melt precrystallized thin films. ${ }^{19}$ Zone casting, in contrast, involves the deposition and evaporation of OSC solution, which flows through a nozzle that is passed over a (typically) temperaturecontrolled substrate. The solution is thus continuously fed to the solution droplet as it is dragged beneath the nozzle.

2.3.3 Hollow-pen writing. Also known as "capillary pen printing," this method makes use of the principles behind the common ink pen. Just as a normal pen deposits a solution of pigments onto paper, hollow pen writing instead uses OSC solutions that can be deposited and patterned on a desired substrate. Typical implementations of this method rely on motorized control to move the vertically mounted pen in all three directions. Normal, commercially available ink pens can be readily adapted for this process.

2.3.4 Blading. There are a variety of so-called "blading" techniques including doctor blading, bar coating, and knifeover-edge methods, and the differences between them are often vague. A solid substrate - a knife's edge, for example - is passed over a solution reservoir, leaving behind a uniform wet layer, after which solvent evaporation is allowed to occur, aided or unaided. In some cases, this process is performed manually, although many are mechanically controlled. As its name suggests, bar coating uses a cylindrical bar that is either smooth $^{20}$ or patterned with grooves or wrapped with wires. ${ }^{21,22} \mathrm{~A}$ key difference between these blading methods and other similar ones is that the entire solution reservoir is typically exposed to the ambient during coating. The coating speed is generally higher than in other techniques, up to of tens of $\mathrm{cm}$ $\mathrm{s}^{-1} \cdot{ }^{23}$ As a facile way to simply spread a solution over a substrate, blading methods have been adapted to roll-to-roll processes $^{\mathbf{2 4 , 2 5}}$ and are amenable to the production of complex, multi-layered organic devices. ${ }^{26,27}$

Edge-casting is similar to other blading techniques in that a solution is simply coated over the substrate by the motion of solid surface above the droplet. Developed by Soeda et al., this method uses a flat, rectangular edge of a coating blade and can produce single-crystalline thin films by using slow solvent evaporation. ${ }^{28}$ A blade is moved at speeds on the order of tens of micrometers per second while sustaining a solution droplet in the gap between the rectangular blade and the substrate, which in ref. 28 was chosen to be $200 \mu \mathrm{m}$. The rectangular solution droplet was continuously fed with fresh solution so that the overall volume remained constant. High boiling point solvents $\left(180\right.$ and $206^{\circ} \mathrm{C}$ ) were used with a moderate substrate temperature $\left(80{ }^{\circ} \mathrm{C}\right)$ to deposit the films. To our knowledge, this technique has not been applied to OSC systems other than the ones from the first report, but the ability to form single crystals is quite attractive.
2.3.5 Slot-die coating. The use of slot dies for coating processes is often associated with the extrusion of polymers and paint coatings. Very simply, an orifice (the slot) permits the flow of material through a shaping device (the die) onto a moving substrate below. Slot-die coating is heavily used in industry and has been adapted to roll-to-roll processes. ${ }^{29}$ Curtain coating, for example, is a slot-die process where an uninterrupted stream of liquid material coats continuous substrate sheets, like photographic film, or discrete three-dimensional objects, like ice cream bars and doughnuts.

The combination of slot-die schemes with roll-to-roll processing has mostly been applied to solar cells ${ }^{30,31}$ and lightemitting diodes ${ }^{32}$ so far. The processing parameters that affect the previously discussed methods like zone casting and dipcoating are applicable here as well.

2.3.6 Solution shearing. Solution shearing is a highly versatile coating technique where a movable top shearing blade holds an OSC solution droplet above a temperature-controlled substrate. $^{33,34}$ The blade is moved relative to the substrate at a fixed speed, exposing the solution meniscus and allowing for solvent evaporation and the deposition of aligned thin films. Compared to other blading techniques, the solution droplet is covered by the blade so that solvent evaporation is confined to only the edges of the droplet. Because of the kinetic nature of the method, previously unobserved metastable molecular packing motifs - so-called "lattice-strained" crystal structures were successfully formed using this method. ${ }^{35}$ The potential to tune molecular packing is valuable, given that altering the intermolecular $\pi-\pi$ stacking distance between OSCs can dramatically enhance charge transport. ${ }^{36-38}$

A modified solution shearing technique developed by Diao et al. recently demonstrated the deposition of organic semiconductor single crystals. ${ }^{39}$ Named "fluid-enhanced crystal engineering”, or FLUENCE, the method introduced two features to rationally control solute nucleation and crystal growth during deposition: (1) a shearing blade patterned with pillars to induce mixing within the sheared solution droplet and (2) a selectively wetting substrate with specially designed shapes. In the latter, the coffee-ring effect ${ }^{40}$ was used to induce nucleation, after which growing crystallites were filtered out so that only one could continue to grow. Mixing within the solution droplet served to reduce the mass transport limitations during crystallization.

\subsection{Printing}

The term "printing" is somewhat loosely defined, and while some may use the word to refer to a very specific type of deposition process, for the purposes of this review we categorize printing processes as those that are amenable to large-area deposition with spatial deposition control and that do not primarily rely on meniscus-driven coating.

2.4.1 Brush painting. Much like hollow pen writing, brush painting draws inspiration from the common usage and applications of brushes to paint or coat solutions or suspensions of pigments. A typical brush is simply dipped into an OSC solution and brushed across the target substrate to coat the surface. In the deposition of polymer OSCs, it was found that 
compared to spincoating, brush painting enhanced the alignment of the polymer chains, suggesting that the presence of two liquid-solid interfaces - the solution/substrate and the solution/brush - allowed for the effective exertion of shear stress throughout the polymer solution. ${ }^{\text {11,42 }}$ This contrasts with spincoating as well as other meniscus-based methods, where the exposed solution surface is a free interface.

2.4.2 Stamping. The use of polymer stamps for the deposition of OSC thin films has been studied and implemented in a variety of contexts. Stamps made of elastomeric poly(dimethylsiloxane) (PDMS) and fluoropolymers have been used to either facilitate solvent evaporation (through absorption into the stamp ${ }^{43}$ or reduce it, ${ }^{44}$ respectively. Because polymer molds can be patterned during their fabrication, features can be designed, in turn, to pattern OSC thin films. Sometimes referred to as a "lithographic" process, stamping spatially confines OSC solutions under ${ }^{45,46}$ or between ${ }^{47}$ protrusions to simultaneously facilitate patterning and controlled solvent evaporation. In some reports, plastic pillar arrays were used, ${ }^{11}$ and patterned silicon molds have been shown to align conjugated polymers. ${ }^{48}$

2.4.3 Inkjet printing. Inkjet printing is a mature research area and has many commercial and scientific applications. It involves the ejection of a jet of ink from a chamber via a piezoelectric or thermal process and the deposition of the soformed droplet onto a desired substrate. Parameters such as ink viscosity, ink surface tension, and substrate surface energy are crucial for the effective ejection and deposition of droplets, and a delicate balance among processing parameters must be struck so that fluid momentum transfer and droplet spreading are precisely controlled. ${ }^{49,50}$ After contacting the substrate, droplets are then allowed to dry like in normal dropcasting methods. The printing of polymer solutions, for example, to form precisely patterned arrays is relatively complex, requiring an understanding of the fluid flow involved. ${ }^{51}$ In many cases, inhomogeneities in the dried OSC films arise from differential solvent evaporation or surface tension gradients in mixed solvent systems. Despite some of these complexities, precise control of these variables have allowed for the deposition of high-performing OSC films. ${ }^{52-54}$

2.4.4 Spray coating. Similar to inkjet printing, spray coating deposition operates by ejection of solution droplets from a nozzle. However, in the latter, the small droplets are formed by aerosolization with an inert carrier gas to coat a substrate. For this method, the spray nozzle shape and size, the atomizing gas pressure, and key solution properties like surface tension and viscosity are highly relevant processing parameters in addition to typical ones like temperature and concentration. ${ }^{55}$ Solution droplets typically hit the substrate and dry rapidly, or in some cases, they form very thin, contiguous wet films. Spray coating setups can be controlled either manually or digitally with motorized manipulators. Standard spray coating methods have been used to fabricate organic solar cells ${ }^{56}$ and photodiodes, among others. ${ }^{57}$

Several variations of the basic spray coating technique have been used for organic electronics applications. For example, multiple spray nozzles have been used in sequence to deposit different OSCs in a layer-by-layer fashion..$^{58}$ Bulk heterojunction organic solar cells ${ }^{59}$ and LEDs ${ }^{60}$ have been fabricated using an evaporative spray technique, whereby very dilute solutions are first aerosolized into a heated chamber and then funnelled through a nozzle into a second chamber containing the target substrate. ${ }^{61}$ Ultrasonic spray nozzles have been used to atomize solutions to droplets on the scale of micrometers, as well as to prevent solution clogging within the nozzle. ${ }^{62-64}$ Lastly, electrospray deposition, where electricity is used to atomize the solution, was shown to effective for the selective deposition of organic materials on metal surfaces. ${ }^{65}$

\section{Morphology control strategies for solution processing}

As summarized in the previous section, recent years have witnessed rapid progress in the development of solution processing methods towards achieving the vision of low-cost, highthroughput, large-area fabrication of organic electronics. A key challenge in the area of solution-processed organic electronics lies in the precise control of thin film morphology during solution deposition. The critical role of thin film morphology in charge transport has been detailed in recent reviews. ${ }^{66-70}$

There has been a long-held perception that most OSCs, when solution deposited, exhibit lower charge carrier mobilities than their vapor-deposited single crystal forms. In the last few years, such a perception has been frequently challenged by the rapid development in morphology control methods during solution processing, such as in the case of 6,13-bis(triisopropylsilylethynyl) pentacene (TIPS-pentacene) (1-73 $^{713}$ and dioctylbenzothienobenzothiophene (C8-BTBT). ${ }^{17,53}$ Thanks to the unique characteristics of solution processing methods, new avenues have been explored for controlling thin film morphology that are not easily implemented during vapor deposition, if at all possible. These new strategies include, but are not limited to, controlling the fluid flow, ${ }^{72,74}$ tuning the solvent composition by addition of an antisolvent or soluble additives, ${ }^{2,53,75}$ controlling evaporation rate using asymmetric patterns, ${ }^{7,53}$ contact line engineering, ${ }^{72}$ alignment control via meniscus guide, ${ }^{46,76}$ etc. These recently developed morphology control strategies will be discussed in the following sections, which pertains to solutionprocessed organic field-effect transistors. The discussion will focus on three aspects: control of nucleation, crystal growth, and domain alignment, with special emphasis on methods that exploit the unique characteristics of solution processing.

\subsection{Control of nucleation}

Crystallization of OSCs is composed of two steps, nucleation and crystal growth. Nucleation involves overcoming of a free energy barrier and is intrinsically stochastic. ${ }^{77,78}$ Such properties often lead to random distribution of domain boundaries and domain sizes. This issue is not unique to solution processing and is also observed during vapor deposition. It is particularly important to control nucleation for the fabrication of single crystal arrays. Thus far, there have been relatively few methods reported on the control of OSC nucleation from solution, as summarized below. 


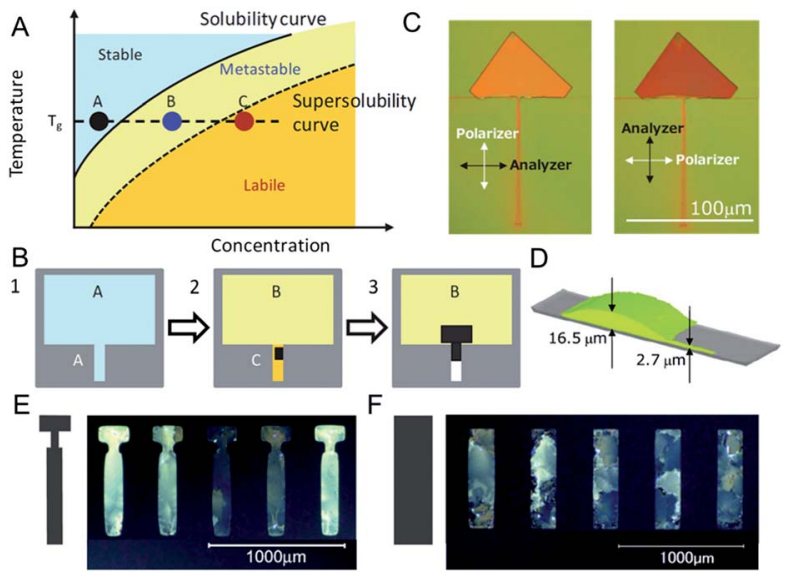

Fig. 2 Nucleation controlled by solution volume asymmetry. Example 1 (A-D). (A) Solubility diagram corresponding to (B). (B) Schematic showing that nucleation occurs where solution volume is much lower. (C) Optical microscopy image of the resulting single crystals of C2PhPXX under cross-polarizers. (D) Calculation results of solution droplet shape. Example $2(E-F)$. Comparison of ink-jet printed C8-BTBT thin film morphology with (E) and without (F) nucleation control region. Images are adapted with permission from ref. 53 (๔ 2011 Nature Publishing Group) and ref. 7 (@ 2012 WILEY-VCH Verlag GmbH \& Co. KGaA, Weinheim).

3.1.1 Solution volume asymmetry. The key to controlling nucleation lies with the control over solvent evaporation, which provides the driving force for nucleation during the coating process. One effective approach is to use asymmetric surface patterns to define asymmetric solution volumes. The region with lower solution volume serves as the nucleation control region due to faster solvent evaporation, and therefore faster rate of supersaturation generation. Recently, Goto et al. ${ }^{7}$ reported large single crystal arrays formed by inducing nucleation at patterned, small solution volume regions where solvent evaporation was faster (Fig. 2a-d). The faster solvent evaporation rate in the nucleation control region was verified using computational fluid dynamics simulations. Using this method combined with controlling the solvent vapor pressure during growth, the authors fabricated single-crystal arrays of 3,9-bis(4ethylphenyl)-peri-xanthenoxanthene (C2Ph-PXX). In addition, the authors demonstrated that preferred crystal orientation could be partially induced by narrowing the width of the nucleation control region from $10 \mu \mathrm{m}$ to $5 \mu \mathrm{m}$. Minemawari et $a l .{ }^{53}$ developed a method for controlling nucleation during double-shot ink-jet printing of C8-BTBT single crystals. In this method, asymmetric patterns were also employed to induce nucleation where the solution volume was lower (Fig. 2e and f). When such patterns were employed, the crystal morphology was drastically improved, with a single-crystal yield of approximately $50 \%$. In comparison, only polycrystalline patterns were obtained using simply rectangular shaped patterns of various aspect ratios, wherein nucleation primarily occurred from the edges.

3.1.2 Contact line curvature. Another approach to control solvent evaporation is tuning of the contact line curvature. Inspired by the mechanism behind the coffee ring effect, ${ }^{\mathbf{4 0}}$ we recently introduced a new strategy for controlling nucleation using this method using solution shearing as the platform, whereby nucleation is anchored at spots where the curvature of the contact line is the highest (Fig. 3). In this method, the shape of the contact line was modulated by patterning the substrate with solvent-wetting and dewetting regions. The initial parts of the wetting regions are shaped as triangles. As the meniscus passes, the contact line is temporarily pinned at the boundary of the triangles, until nucleation occurs at the sharp tips. The triangular design is also beneficial in that it defines a wedge shaped meniscus that funnels the convective supply of solute towards the tip, which facilitates nucleation anchoring by lowering the nucleation induction time. In addition, the triangles are designed to be asymmetric to eliminate twin boundary formation. Following the triangles is a series of very narrow neck regions in the pattern whose purpose is to arrest the growth of undesired crystallites, which are otherwise difficult to

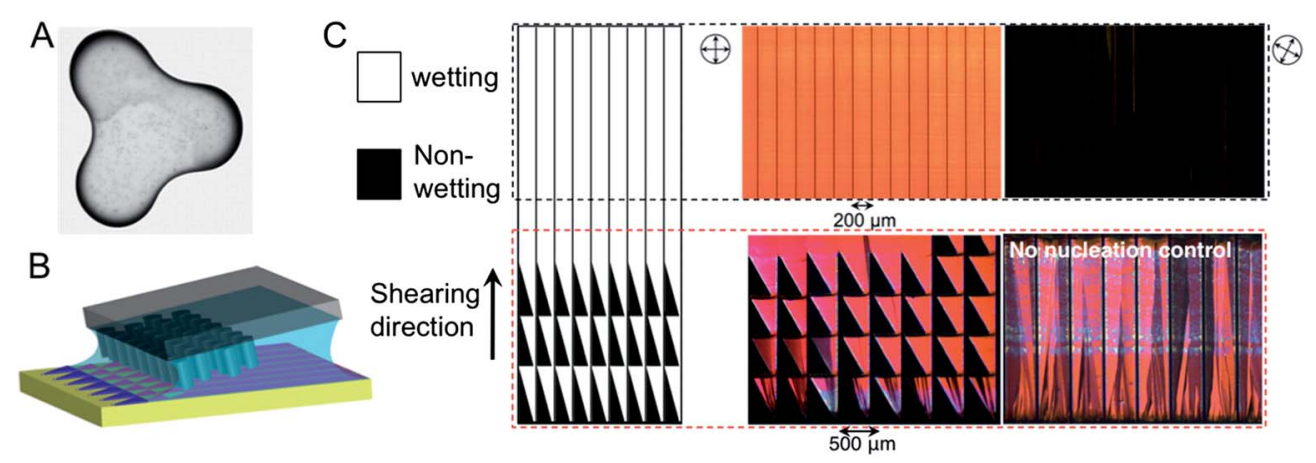

Fig. 3 Nucleation controlled by tuning the contact line curvature. (A) Dependence of coffee ring thickness on contact line curvature. (B) Schematic of FLUENCE (fluid-enhanced crystal engineering) incorporated in solution shearing, wherein this nucleation control concept is implemented. (C) Design of wetting/dewetting zones for nucleation control (left) and the resulting morphology of FLUENCE-printed TIPSpentacene thin films (right). The lower panel compares morphology obtained with and without nucleation control. The upper panel shows highly aligned single-crystalline domains obtained using FLUENCE, which extinguish cross-polarized light at once. All optical images were obtained under crossed polarized light. Images are adapted with permission from ref. 40 (๔ 1997 Nature Publishing Group) and ref. 72 (@ 2013 Nature Publishing Group). 
eliminate simply by nucleation control. When combined with crystal growth control, this method enabled high probability of single-crystalline domains ( $>90 \%$ with $200 \mu \mathrm{m}$ wide patterns, Fig. 3c) for the OSCs tested (TIPS-pentacene and 4T-TMS). This method is expected to be applicable to other deposition processes other than solution shearing, given the generality of curvature-modulated solvent evaporation. One drawback of this method is the relatively long 'runway' before the single-crystal growth 'takes off', which may limit the its use in fine-detail patterning.

3.1.3 Nucleation-inducing agents. In addition to tuning the solvent evaporation rate by designing substrate patterns, nucleation can also be controlled using soluble additives. Such approach has been utilized to enable systematic studies of the impact of domain sizes on charge transport in solvent-vapor annealed TES-ADT thin films. ${ }^{2}$ The understanding of how additives impact nucleation also has implications on dopant design, ${ }^{79}$ whereby dopant molecules come into play as soluble additives during film morphology evolution. ${ }^{73}$ However, the role of dopant on morphology of solution-processed OTFT remains to be further explored.

Recently, two inert additives (DMDBS, BTA; Fig. 4a), originally designed for melt solidification of isotactic polypropylene (i-PP), were shown to effectively induce nucleation of a diverse set of OSCs by Stingelin, Chabinyc and coworkers. ${ }^{75}$ These two

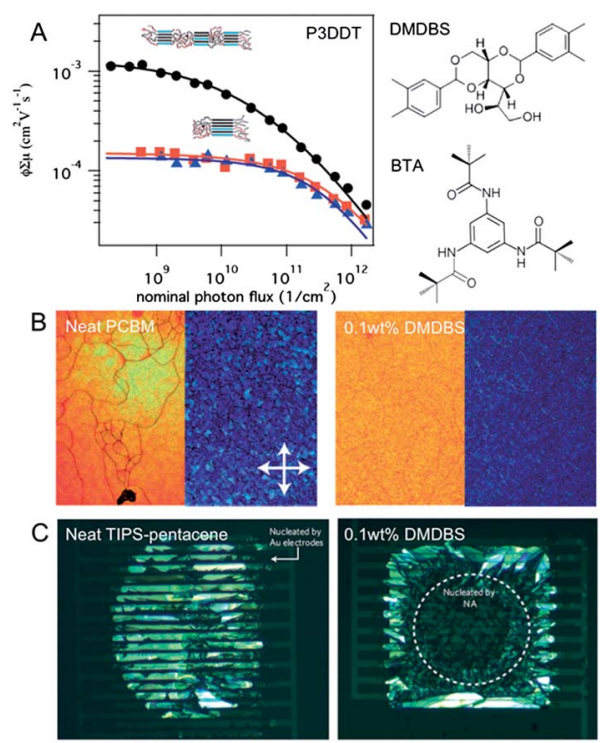

Fig. 4 Nucleation controlled using heterogeneous additives. (A) timeresolved microwave conductivity (TRMC) measurements of yieldmobility product of poly(3-dodecylthiophene) (P3DDT), neat (orange squares) and comprising DMDBS (black circles) or BTA (blue triangles). (B) Transmission optical micrographs of neat PCBM $(99.5 \%$ electronic grade) drop cast at room temperature from chlorobenzene (left), with 0.1 wt\% DMDBS (middle), and subsequently annealed at $180{ }^{\circ} \mathrm{C}$. (C) Crossed-polarized optical micrographs (height: $660 \mu \mathrm{m}$ and width: $820 \mu \mathrm{m}$ ) of an inkjet printed drop onto an OFET (Organic Field Effect Transistor) substrate with a dielectric treated with octadecyltrichlorosilane (OTS): ink comprising no nucleation agent (left); ink comprising a minute amount of DMDBS (right). Figures are adapted with permission from ref. 75 (๔ 2013 Nature Publishing Group). electrically insulating compounds initially dissolve at the molecular level in the molten polymer or solution, and, on cooling or solvent removal, form well-dispersed, nanoscopic surfaces for heterogeneous nucleation of the host material.

The authors found that the presence of the additive DMDBS in polymer P3DDT led to an increase of up to $50 \%$ in carrier yield compared with the neat polymer (Fig. 4a), which is attributed to the creation of a larger interfacial area between crystalline and amorphous domains through heterogeneous nucleation. A minute amount of DMDBS also reduced the domain sizes of annealed PCBM without adversely affecting the electron mobility. This effect was further explored as a patterning method by controlled deposition of the additive. With the nucleation agents, the authors also improved the device yield of bottom-contact TIPS-pentacene devices fabricated via ink-jet printing. The dielectric surface was functionalized with octadecyltrichlorosilane, a self-assembled monolayer shown extensively to improve charge transport in vapor deposited active layers. ${ }^{\mathbf{8 0}}$ However, its low surface energy leads to dewetting of most organic solvents. Upon addition of DMDBS, the area coverage of TIPS-pentacene film greatly improved (Fig. 4c), whereas without the nucleation agent, the crystallization preferentially occurred on the Au electrodes. The yield of bottom contact transistor devices was therefore increased upon addition of the nucleating agent DMDBS. The hole mobility of the transistors was on the order of $10^{-2} \mathrm{~cm}^{2} \mathrm{~V}^{-1}$ $\mathrm{s}^{-1}$.

These examples show that enhanced nucleation using additives can greatly improve the device area coverage and can potentially enable device patterning by placing additives at predefined locations on the substrate. However, the addition of nucleation-inducing agents inevitably leads to reduction in domain sizes, which is often undesirable for transistor applications due to lowered charge carrier mobilities in cases where domain boundaries are rate-limiting - especially in the case of high-angle domain boundaries. ${ }^{81}$ Nonetheless, the reduction in domain size may prove beneficial to organic photovoltaic applications in some cases.

Other methods towards achieving nucleation control in vapor deposition or melt processing methods include the use of patterned rough surfaces, ${ }^{\mathbf{8 2 , 8 3}}$ the use of chevron-shaped patterns for controlling silicon nucleation from its melt during laser annealing, ${ }^{\mathbf{8 4}}$ etc. Nonetheless, there is a very limited number of approaches developed for controlling nucleation of OSCs, largely due to the challenge of attempting to control an intrinsically stochastic process.

\subsection{Control of crystal growth}

Crystal growth control impacts the level of crystallinity/structural perfection, domain size distributions, substrate coverage, OSC-dielectric interface quality, etc., all of which are key to controlling the charge carrier mobility. In the following, we highlight three recently developed strategies for controlling crystal growth: by novel design of antisolvent crystallization, the new concept of flow-assisted crystallization, and solvent vapor annealing. These methods are designed to facilitate growth of 

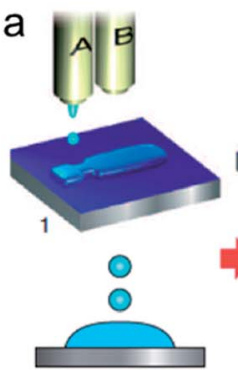

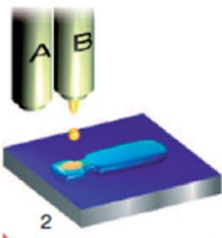

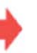

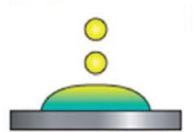

$\Rightarrow$
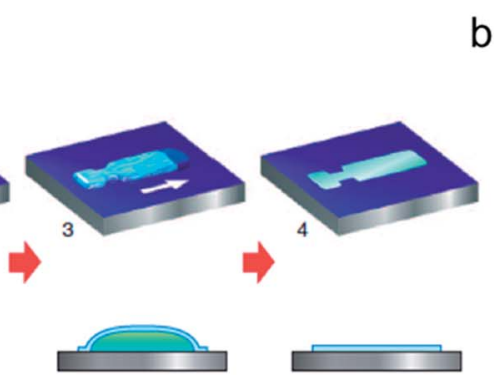

b

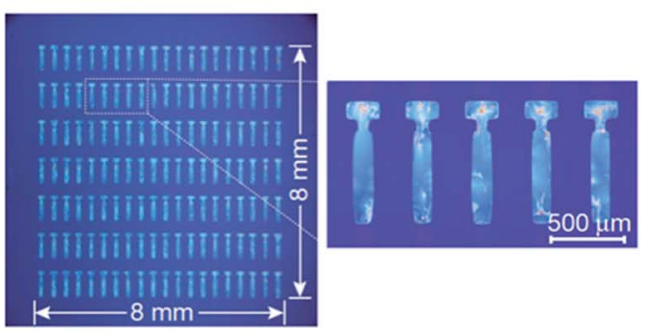

Fig. 5 Crystal growth control via antisolvent crystallization. (a) Schematic of the process. Antisolvent ink (A) is first inkjet-printed (step 1), and then solution ink (B) is overprinted sequentially to form intermixed droplets confined to a predefined area (step 2). Semiconducting thin films grow at liquid-air interfaces of the droplet (step 3), before the solvent fully evaporates (step 4). (b) Micrographs of a $20 \times 7$ array of inkjet-printed C8BTBT single-crystal thin films. Figures are adapted with permission from ref. 53 (๔ 2011 Nature Publishing Group).

large, highly crystalline domains by enhancing molecular mobility or eliminating mass transport limitations with the help of soft interfaces, fluid flow, or solvent vapor.

3.2.1 Antisolvent crystallization. Antisolvent crystallization is widely utilized in the crystallization of pharmaceutical ingredients as an excellent method for achieving controlled and scalable solidification. Hasegawa and coworkers have applied this method on the ink-jetting printing platform for fabrication of C8-BTBT single crystal arrays (Fig. 5). ${ }^{53}$ In this method, an "antisolvent" (a liquid in which a substance is insoluble) is added to the solution of the substance in a solvent that is miscible with the antisolvent. During ink-jet printing, a drop of antisolvent was deposited on a pre-defined area followed by a drop of semiconductor solution. As the antisolvent diffuses into the ink solution, crystallization starts at the solution-air interface, as tiny floating bodies. These mini crystals eventually cover the entire surface the droplet. The solvent then evaporates very slowly, during which time, the creases in the films become
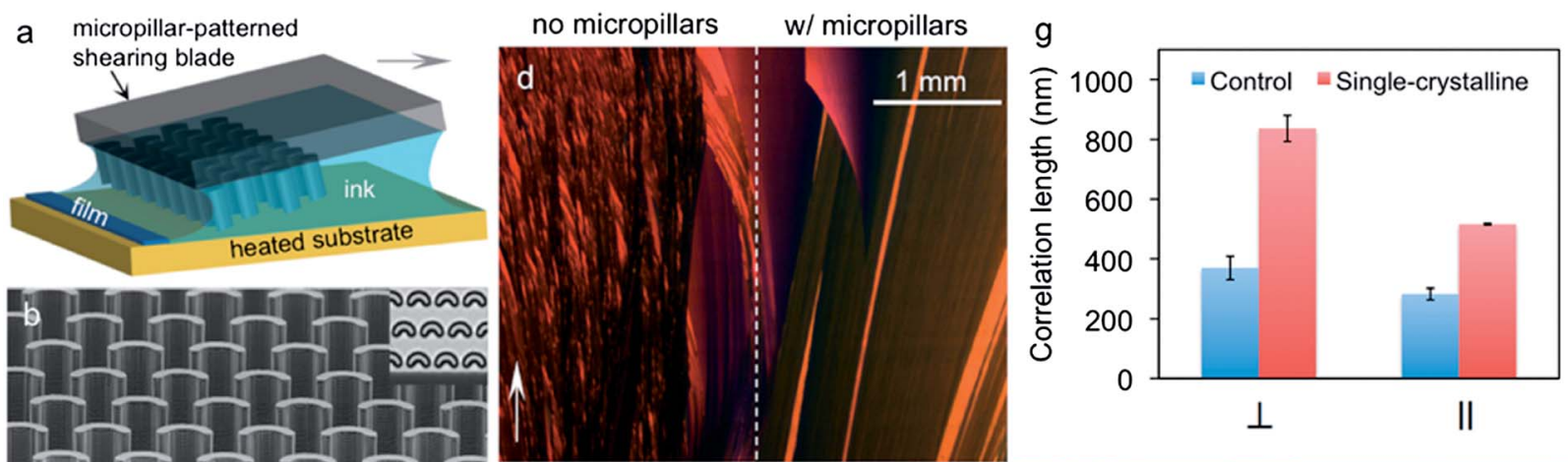

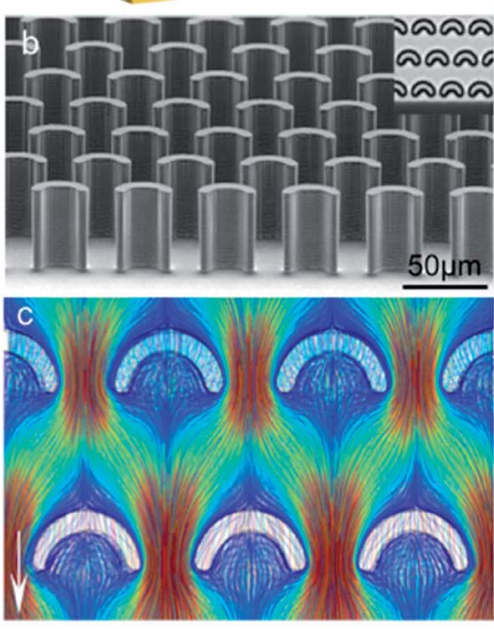

Methodology

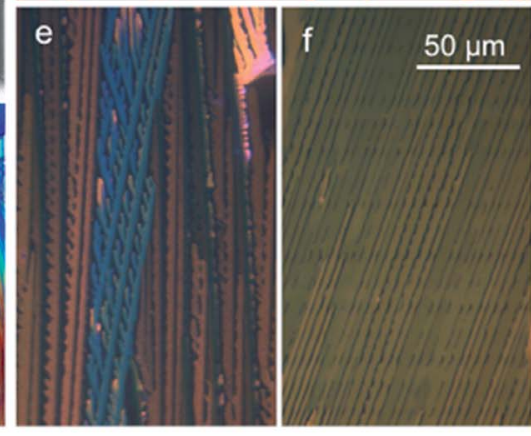

TIPS-Pen

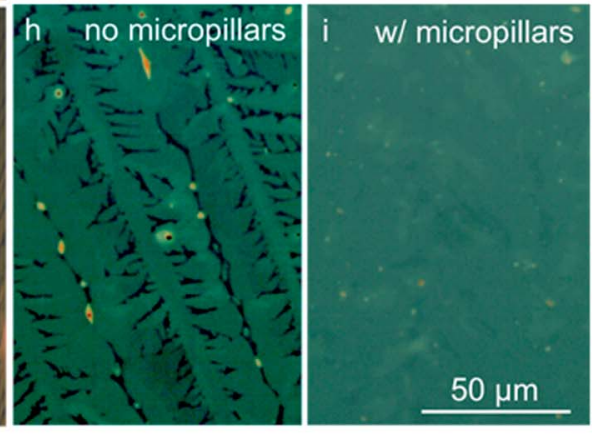

4T-TMS

Fig. 6 Crystal growth controlled using micropillar arrays. (a) Schematic of solution shearing using a micropillar-patterned blade. For clarity, the micropillars are not drawn to scale. The arrow indicates the shearing direction. (b) A scanning electron micrograph of a micropillar-patterned blade. Inset, top view of the micropillars under an optical microscope. The pillars are $35 \mu \mathrm{m}$ wide and $42 \mu \mathrm{m}$ high. (c) Streamline representation of simulated fluid flow around the micropillars. The arrow indicates the flow direction. The streamlines are colour coded to indicate the scale of velocity $\left(\mathrm{mm} \mathrm{s}^{-1}\right.$ ), ranging from 0 (deep blue) to $1.3 \mathrm{~mm} \mathrm{~s}^{-1}$ (dark red). (d-f) Cross-polarized optical micrograph of a TIPS-pentacene film coated from its mesitylene solution with (d, right; f) and without micropillars (d, left; e), at a shearing speed of $0.6 \mathrm{~mm} \mathrm{~s}^{-1} \mathrm{~g}^{-1}$, Comparison of TIPSpentacene coherence length with and without nucleation and crystal growth control along both parallel and perpendicular to shearing direction. (h and i) Comparison of 4T-TMS film morphology with and without using micropillar patterned blade. Figures are adapted with permission from ref. 72 (๔ 2013 Nature Publishing Group). 


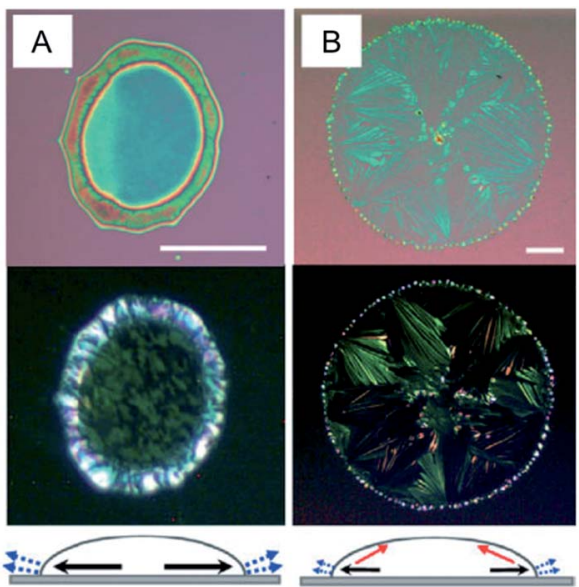

Fig. 7 Crystal growth controlled by inducing Maragoni flow. Optical microscope (OM) and polarized images of ink-jet-printed TIPS_PEN droplets with various solvent compositions: mixed-solvents containing chlorobenzene and 25 vol\% (A) hexane and (B) dodecane (scale bar $=50 \mu \mathrm{m})$. Schematic diagrams of the evaporation-induced flow in a droplet during drying for various solvent compositions are shown under the corresponding images, where the arrows indicate the evaporation of solvent (blue), the outward convective flow (black), and the Marangoni flow (red). Images are adapted with permission from ref. 74 (๔ 2008 WILEY-VCH Verlag GmbH \& Co. KGaA, Weinheim).

smoothed out, resulting in a film adhered tightly to the substrate. The final process of slow solvent evaporation underneath the floating crystal is akin to the solvent vapor annealing process for increasing the domain size and healing the crystal defects. The control of crystal growth, combined with controlled nucleation, led to record high hole mobility (at the time of publication) of top-contact, top-gate devices, the highest of which exceeded $30 \mathrm{~cm}^{2} \mathrm{~V}^{-1} \mathrm{~s}^{-1}$.

3.2.2 Flow-assisted crystallization. Crystal growth defects are commonly observed during rapid solution coating. ${ }^{85,86}$ Mass transport limitations during the coating process often leads to void formation and dendritic growth, both of which hinder efficient charge transport due to charge carrier trapping at the prevalent grain boundaries. To address this issue, we developed a technique that involves patterning of the coating blade with micropillars to enhance mass transport by re-directing the fluid flow (Fig. 6). ${ }^{72}$ With the insight from fluid dynamics simulations, we designed the following patterns. The pillar spacing (period) is sub-100 microns to match the typical domain size in the reference film prepared without using micropillars. The cross-section of the micropillars is crescent-shaped, arching against the flow direction to encourage flow separation from the surface of the micropillars. This design is intended to generate recirculation behind the pillars. The narrow pillar spacing is designed to induce rapid flow expansion following acceleration through the gap, so as to facilitate mass transport in direction transverse to the coating direction where diffusive mass transport dominates. This method was implemented on the solution shearing platform. The use of micropillar-patterned shearing blades significantly improved the thin-film morphology of TIPSpentacene and 4T-TMS. The TIPS-pentacene domain size increased from micron-sized to as large as millimeter-sized, and the dendritic growth was eliminated in both cases. Furthermore, the in-plane coherence lengths of the crystal increased significantly, indicating higher degree of structural perfection. The highest coherence length attained even matched with that of the vapor-deposited rubrene crystal. These structural features, together with controlled nucleation and optimized molecular packing boosted the hole mobility of TIPS-pentacene to higher than $10 \mathrm{~cm}^{2} \mathrm{~V}^{-1} \mathrm{~s}^{-1}$, the highest reported so far for this extensively studied material.

In addition to directing the fluid flow using structured printing blades, another elegant approach has been developed by Cho and coworkers wherein Marangoni flow was induced using mixed solvents to overcome the coffee ring effect frequently observed during inkjet printing. ${ }^{74}$ Marangoni flow is a type of flow caused by a surface tension gradient, and the
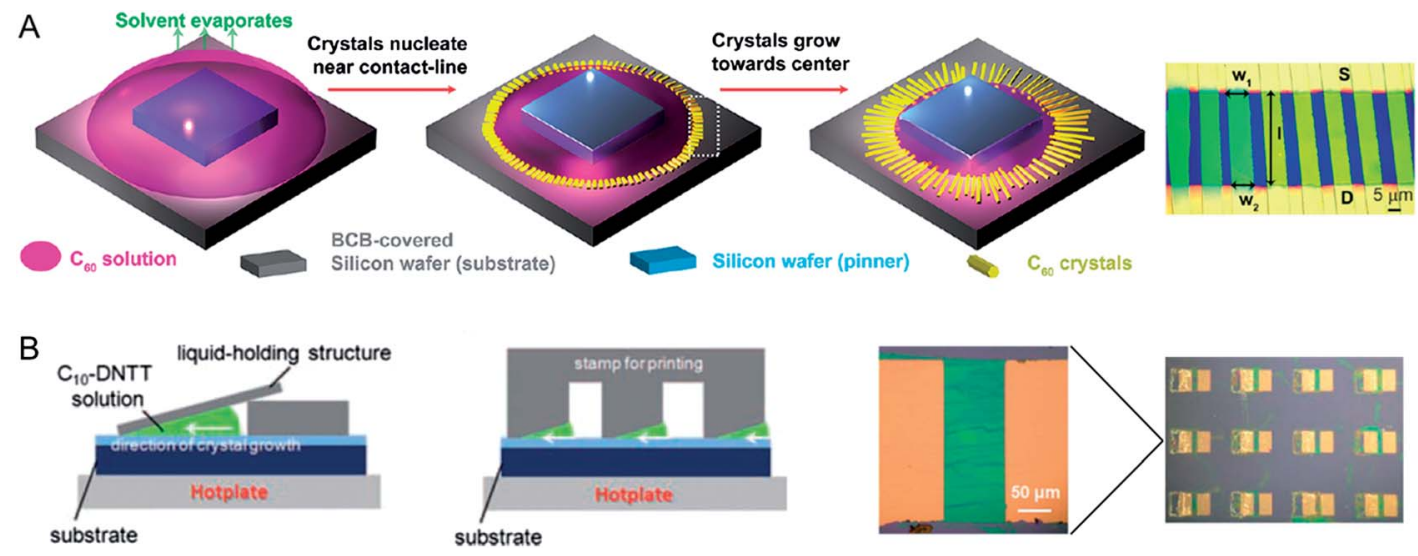

Fig. 8 Aligned crystal arrays using static meniscus guide. (A) An organic semiconductor droplet pinned by a silicon wafer. As the solvent evaporates slowly, the crystals of the organic semiconductors nucleate near the contact line of the droplet. Subsequently, the nuclei grow along the receding direction (toward the center) of the droplet. Optical microscopy images showing C60 crystals between source and drain electrodes. (B) Methods of fabricating the crystallized high-mobility C10-DNTT films on substrates using tilted stamps and resulting film morphology. Images are adapted with permission from ref. 46 (C) 2012 American Chemical Society) and ref. 76 (@2011 WILEY-VCH Verlag GmbH \& Co. KGaA, Weinheim). 


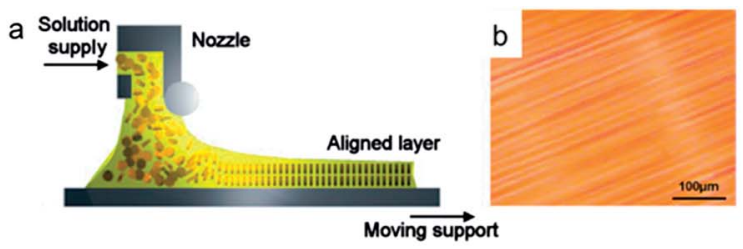

C

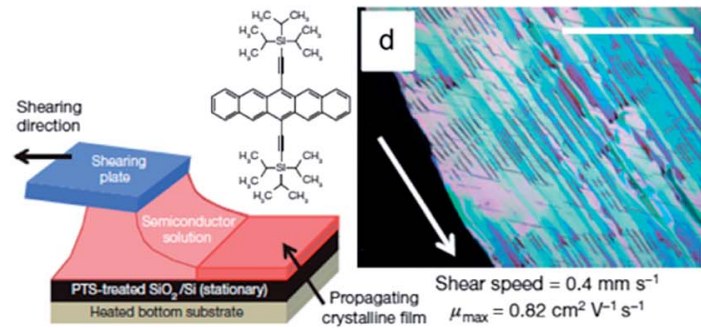

e

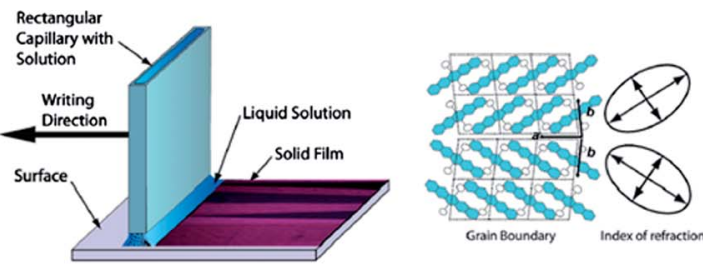

f

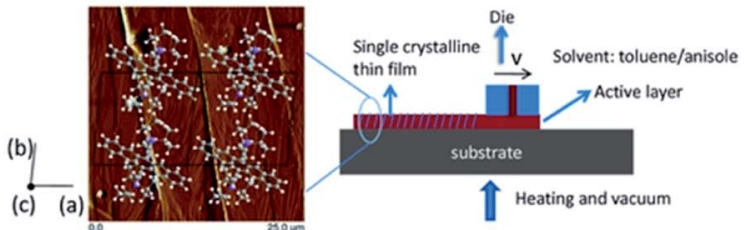

Fig. 9 Aligned crystal arrays using moving meniscus guide. ( $a$ and b) Schematic of zone casting and the resulting film morphology of dodecyl-substituted HBC derivative $\left(\mathrm{HBC}-\mathrm{C}_{12}\right){ }^{99}$ (c and d) Schematic of solution shearing and the resulting film morphology of TIPS-pentacene. ${ }^{33,71}$ (e) Schematic of hollow pen writing and the twin domains of TIPS-pentacene as a result. ${ }^{86}$ (f) Schematic of slot-die coating and the AFM image of the film with molecular structure superimposed. ${ }^{101}$ Images adapted with permission from ref. 99 (@ 2005 WILEY-VCH Verlag GmbH \& Co. KGaA, Weinheim), ref. 3 (๔ 2011 Nature Publishing Group), ref. 86 (๔ 2012 AIP Publishing LLC) and ref. 101 (๔ 2013 WILEY-VCH Verlag GmbH \& Co. KGaA, Weinheim).

direction of the flow points towards higher surface tension regions. The convective flow that transports the solute to the contact line can be counterbalanced or enhanced, depending on the direction of the Marangoni flow. When adding a cosolvent of higher boiling point and lower surface tension, a Marangoni flow with a direction opposite that of the convective flow can be induced. On the other hand, the Marangoni flow can also enhance the convective flow if a low boiling point solvent with high surface tension is added as the minor component. Based on this strategy, the evaporation-induced flow in droplets can be controlled by varying the composition of the solvent mixture. The authors demonstrated this concept during ink-jet printing of TIPS-pentacene using chlorobenzene as a major solvent. When adding 25 vol\% hexane (with lower boiling point and lower surface tension than the major solvent) as a minor solvent, the coffee-ring effect was found enhanced due to increased outward convective flow. When adding $25 \mathrm{vol} \%$ dodecane (with higher boiling point and lower surface tension than the major solvent), the coffee-ring effect was significantly reduced instead, thanks to the inward Marangoni flow (Fig. 7). Using this approach, both the film uniformity and the out-ofplane alignment were markedly improved, resulting in improved charge carrier mobility from $1.18 \times 10^{-3}$ (hexane) to $0.12 \mathrm{~cm}^{2} \mathrm{~V}^{-1} \mathrm{~s}^{-1}$ (dodecane). Although currently only demonstrated with ink-jet printing, this approach may be educational to other printing methods for alleviating meniscus pinning, which causes uneven film morphology at times.

3.2.3 Solvent vapor annealing. The solvent vapor annealing method, first applied to organic semiconductors by Bulovic and coworkers for growing tris(8-hydroxyquinoline)aluminum ( $\mathrm{Alq}_{3}$ ) crystals,${ }^{87}$ is a simple yet powerful post-processing technique for drastically improving film morphology and even for obtaining single crystals following vapor deposition or spin coating. Using this method, spin-coated amorphous films of triethylsilylethynyl anthradithiophene (TES-ADT) was transformed into polycrystalline films with domain sizes of several hundred microns. ${ }^{88}$ As a result, the hole mobility in bottom contact bottom gate devices increased by two orders of magnitude to 0.1 $\mathrm{cm}^{2} \mathrm{~V}^{-1} \mathrm{~s}^{-1}$. Solvent selection was found to be critical in this method, which influences both the partitioning of solvent molecules in the active layer and the proper substrate wetting during vapor annealing. ${ }^{\mathbf{8 8}}$ In addition, substrates were also found to play an important role in controlling crystal growth. ${ }^{\mathbf{8 9 , 9 0}}$ Crystal size and morphology of C8-BTBT was improved when the film was solvent vapor annealed on poly(methyl methacrylate) (PMMA) substrate, but not on $\mathrm{SiO}_{2}$ substrates. ${ }^{90}$ When the vertically phase-separated film of C8-BTBT/PMMA was prepared in one step during spin coating, subsequent vapor annealing yielded even larger C8-BTBT single crystals, and correspondingly, the hole mobility measured in top contact and bottom gate geometry reached as high as $9 \mathrm{~cm}^{2} \mathrm{~V}^{-1} \mathrm{~s}^{-1}$. However, the alignment of C8-BTBT single crystals remains random in this method, yielding a wide distribution of charge carrier mobilities. Recently, Loo and coworkers have elegantly shown that by tuning the surface energy of the substrates, crystal growth in asspun TES-ADT films during vapor annealing can be directed along pre-specified paths over arbitrarily large areas, therefore controlling the grain orientation and achieving patterning during solvent vapor annealing. ${ }^{89}$ To shed light on the solvent vapor annealing process, in-situ techniques based on quartz crystal microbalance with dissipation (QCM-D) and grazing incidence X-ray diffraction have recently been developed to monitor both solvent mass uptake and changes in the mechanical rigidity of the film during solvent vapor annealing of spin-cast films of TIPS-Pentacene. ${ }^{91}$ Through this study, the important role of solvent vapor pressure on the final film morphology and device performance was elucidated.

Other methods for controlling crystal growth of solutionprocessed organic semiconductors include templated growth using thiol-functionalized electrodes to increase crystalline grain sizes, ${ }^{\mathbf{9 2}}$ epitaxial growth of polymer films templated by solidified solvent crystals from undercooled solution, ${ }^{\mathbf{9 3 - 9 5}}$ enhanced film morphology, crystallinity or interface quality using external fields (ultrasound, ${ }^{\mathbf{9 6}}$ electric field, ${ }^{\mathbf{9 7}}$ vibration $^{98}$ ), etc. 


\subsection{Control of in-plane alignment}

The impact of in-plane alignment of crystalline domains on charge carrier mobility has been fully demonstrated by studies on charge transport anisotropy. Within a single-crystalline domain, the mobility anisotropy is frequently on the order of 110. ${ }^{102-105}$ Across a polycrystalline film, the mobility anisotropy can range from a few times to several orders of magnitude depending on the nature of grain boundaries. ${ }^{\mathbf{8 1 , 8 6 , 9 3 , 1 0 6}}$ Such sensitivity of charge transport to in-plane crystal orientation highlights the importance of controlling domain alignment. Over the past decade, many techniques have been developed to achieve in-plane alignment. ${ }^{68,70}$ This review will mainly focus on alignment methods newly developed within the past five years, which took advantage of the unique characteristics of solution processing, such as molecular assembly in a moving meniscus and under a flow field.

3.3.1 Alignment using meniscus guide. The meniscus, in particular the solution-substrate-vapor triple phase contact line, is where a dynamic molecular assembly process takes place in the majority of the solution processing methods (when film deposition occurs in the evaporation regime ${ }^{\mathbf{1 0 7}}$ ). By guiding the meniscus in a particular direction, alignment of crystalline domains has been achieved using a variety of methods (Fig. 8 and 9). As diverse as these methods may appear, the mechanisms of achieving film alignment are similar in that the direction of the convective flow towards the meniscus front is guided using static or moving surfaces. The underlying force directing the meniscus motion can be attributed to the capillary force.
Fig. 8 highlights two methods in which static meniscus guides were employed during solvent evaporation: drop-pinned crystallization ${ }^{76}$ and edge casting. ${ }^{46}$ In the first method, the OSC solution droplet is pinned using a small piece of silicon wafer. The authors also pointed out another important requirement for achieving alignment: high nuclei density (i.e., high solution concentration). Using this method, the authors have fabricated a variety of organic thin film transistors and inverters and demonstrated high charge carrier mobilities, enabled by improved alignment and crystal-dielectric interface. ${ }^{10,76}$ Particularly, aligned C60 single-crystal arrays prepared using this method yielded unprecedented electron mobilities as high as 11 $\mathrm{cm}^{2} \mathrm{~V}^{-1} \mathrm{~s}^{-1}$. In the method of edge casting, the meniscus is guided using inclined surfaces that cover the solution droplet during solvent evaporation (Fig. 8b). Using this method, the authors demonstrated aligned crystal arrays of 2,9-alkyldinaphtho[2,3- $\left.b: 2^{\prime}, 3^{\prime}-f\right]$ thieno[3,2- $\left.b\right]$ thiophene (C10-DNTT) with highest hole mobility exceeding $10 \mathrm{~cm}^{2} \mathrm{~V}^{-1} \mathrm{~s}^{-1}$. Both methods offer additional advantages besides alignment, such as patterned deposition over large area and the ability to handle low solubility organic semiconductors.

In recent years, a number of industry-compatible, large-area solution coating techniques (Fig. 9) have been developed for fabrication of aligned organic semiconductor thin films (mostly polycrystalline), such as zone casting, ${ }^{99}$ solution shearing, ${ }^{33,71-73}$ hollow-pen writing ${ }^{86,100}$ and slot-die coating, ${ }^{101}$ which we have surveyed in Section 1. A common feature of these methods is that the meniscus is guided by a moving surface, which can be a

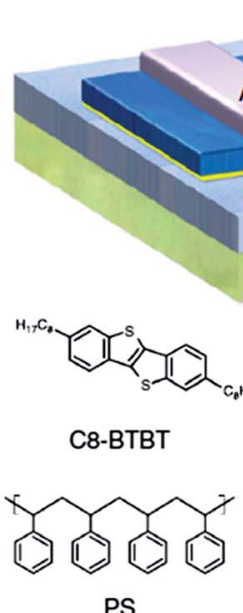

PS

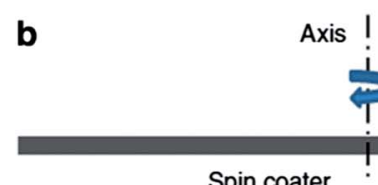

Spin coater
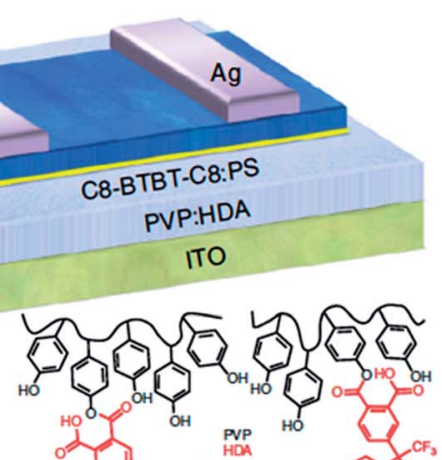

PVP:HDA

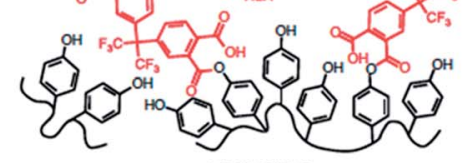

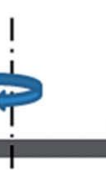

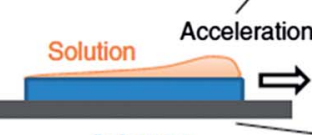

Substrate

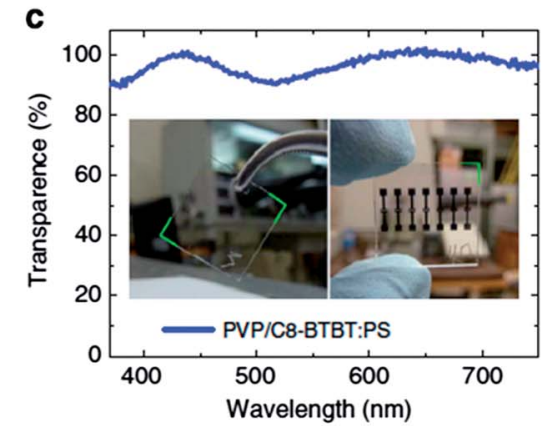

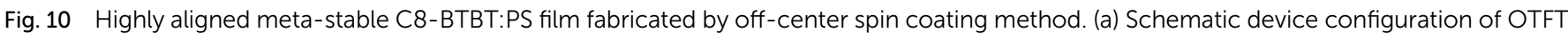

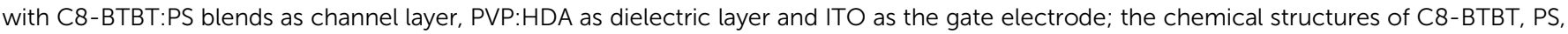

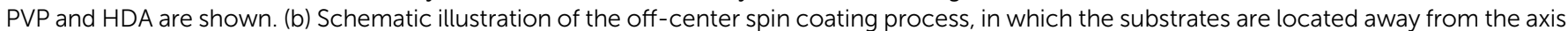

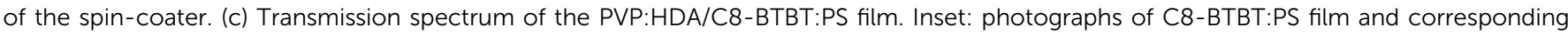

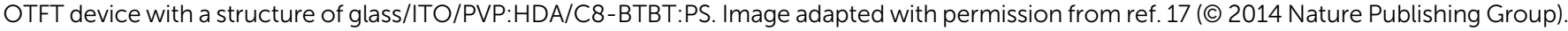


provided by an ink-feeding head in some cases. One major advantage of moving meniscus guides as compared to static ones lies with the wide tunability of coating/printing speeds, which offers accessibility to a range of morphology features, from aligned polycrystalline thin films to spherulite formation. ${ }^{71,100,108}$ It also offers accessibility to nonequilibrium molecular packing, which was proved beneficial to enhancing charge carrier mobilities in the case of TIPS-pentacene. ${ }^{71,72}$ In fact, roll-to-roll printing methods frequently employ moving meniscus guides as well, ${ }^{25,31}$ and in those cases the fundamental molecular assembly processes are similar to those in the methods mentioned above.

3.3.2 Alignment using non-contact forces. In addition to using static or moving meniscus guide, film alignment has also been achieved using non-contact, built-in forces specific to each solution processing method. These forces include gravitational force during dip-coating ${ }^{85,106}$ and dropcasting on tilted substrates, ${ }^{\mathbf{1 0 9 , 1 1 0}}$ drag force exerted by gas flow during dropcasting, ${ }^{6}$ etc. Use of other external forces not unique to solution processing is covered elsewhere. ${ }^{70}$

Recently, Bao, Huang, and coworkers described the growth of highly aligned C8-BTBT from a blended solution of C8-BTBT and polystyrene using an off-center spin-coating method. ${ }^{17}$ In this method, the film alignment was achieved by guiding the meniscus motion using centrifugal force by placing the substrate in an off-center position (Fig. 10). The resulting C8BTBT film was highly aligned and exhibited a metastable crystal packing. These morphological features combined with improved dielectric interface attained via vertical phase separation led to an ultrahigh hole mobility up to $43 \mathrm{~cm}^{2} \mathrm{~V}^{-1} \mathrm{~s}^{-1}(25$ $\mathrm{cm}^{2} \mathrm{~V}^{-1} \mathrm{~s}^{-1}$ on average), which is the highest value reported to date for all organic molecules.

3.3.3 Post-formation alignment using flow fields. Another method to achieve alignment over a large area is to orient existing single crystals carried in the solution using flow field. ${ }^{\mathbf{1 1 1 - 1 1 4}}$ The crystals aligned in this fashion are often highly anisotropic in their shape, such as in the form of microwires. For example, a filtration-and-transfer (FAT) method was developed, which allows for efficient alignment of organic wires with controllable density over a large area (Fig. 11). ${ }^{115}$ Briefly, microwires synthesized by the non-solvent nucleation method are dispersed in a poor solvent such as methyl alcohol or ethyl alcohol. Microwires are aligned by fluid flow through a mask in a modified, simple vacuum filtration setup. Individual singlecrystalline PTCDI microwire-OFETs showed electron mobilities up to $1.4 \mathrm{~cm}^{2} \mathrm{~V}^{-1} \mathrm{~s}^{-1}$, among the highest solution-processed $n$ channel organic semiconducting wire devices at the time when this research was conducted, while high-density microwireOFETs only exhibited mobilities around $0.14 \mathrm{~cm}^{2} \mathrm{~V}^{-1} \mathrm{~s}^{-1} .^{115}$ Post-alignment using flow field is a promising method that exploits the unique characteristics of solution processing. Similar methods have been widely utilized for aligning carbon nanotubes, ${ }^{116,117}$ single macromolecule chains ${ }^{118,119}$ etc., and can inspire new methods to align crystals in OSC applications.

Besides in-plane alignment, out-of-plane alignment is desirable for both OTFT and OPV applications. The readers are referred to recent reviews covering this topic..$^{\mathbf{7 0 , 1 2 0}}$

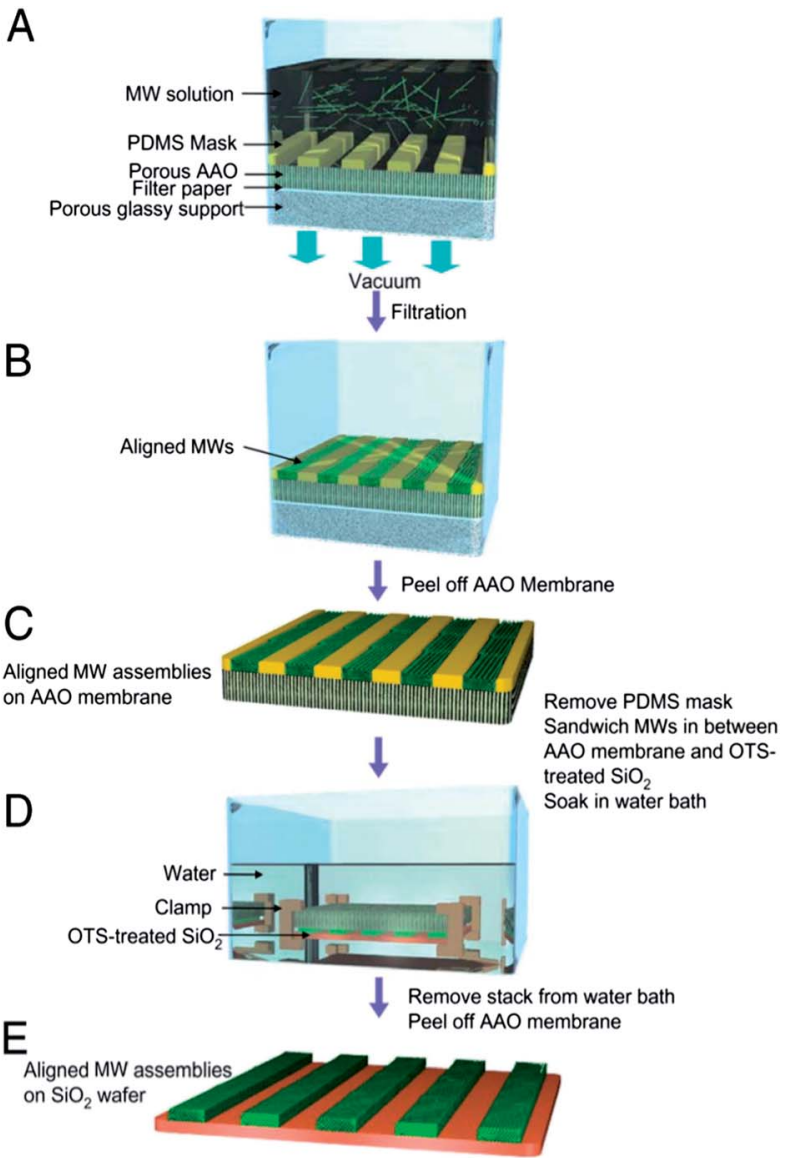

Fig. 11 Schematic diagram for filtration and transfer (FAT) alignment of organic microwires. (A) FAT alignment apparatus loaded with the microwire dispersion. A PDMS mask with open-stripe patterns is placed on a porous AAO membrane. The microwire dispersion is filtered by applying vacuum. (B) Microwire assemblies reside exclusively inside the stripe patterns of the PDMS mask after filtration. The alignment of microwires along the stripe patterns is improved substantially as the pressure difference across the filter stack is increased. The density of the aligned microwires inside a single stripe pattern can be controlled by simply changing the concentration of the MW dispersion. (C) AAO membrane covered with patterned microwires inside the PDMS mask. (D) Illustration for the transfer of the aligned MW patterns from an AAO membrane to a desired wafer substrate in aqueous medium. Microwires selectively adhere to the OTS-treated $\mathrm{SiO}_{2}$ as water diffuses through the pores of the AAO membrane. (E) Aligned microwires were transferred onto the wafer. Images adapted with permission from ref. 115 (C) 2009 National Academy of Sciences, USA).

\section{Conclusions and outlook}

The rapid advancement of thin film solution processing methods and morphology control strategies during recent years has brought us closer to the bright future promised by organic electronics applications. It is clear that choosing the appropriate processing method and deposition parameters is at least as important as the choice of organic semiconductor material. In order to fully exploit the potential of modern solution processing methods for organic semiconductors, a thorough understanding and control of the complex nucleation and 
crystal growth processes during thin film formation is indispensable. During solution processing, the crystal formation occurs in a multi-phase environment. Nucleation and crystal growth are critically influenced by the pairwise interactions between solute, solvent, and substrate, by the presence of phase boundaries, and by the multi-phase mass and heat transport processes. All these parameters jointly determine the morphology of organic semiconductor thin films, controlling which is key to achieving the desired electronic properties. Based on the understanding of these complexities during solution processing, strategies have been devised for tuning the morphological parameters relevant to device performance (grain sizes, grain boundaries, grain orientations, crystallinity, etc.) by controlling the fluid flow, tuning the solvent composition using antisolvent and additives, controlling evaporation rate using asymmetric patterns, or engineering the triple-phase contact line. Some of these strategies have been utilized for fabricating solution-processed single crystal arrays over a large area, which enabled record-setting charge carrier mobilities. $^{\mathbf{1 7 , 3 9 , 5 3}}$ Looking ahead, however, many challenges still remain, such as better control of defect densities, controlled formation and characterization of high quality OSC-dielectric interfaces, a quantitative understanding of the role of molecular packing on electronic properties, and morphological evolution in the context of high resolution printing where new phenomenon arise at sub-micron lengths scales, just to name a few. However, the numerous worldwide research efforts today (of which we could only capture a few in this review) and the large variety and steadily increasing quantity of reports describing new and innovative solution deposition methods for organic (plastic) electronics materials give confidence that most of these challenges will be successfully met in the near future.

\section{Acknowledgements}

S.C.B.M., Z.B. and Y.D. acknowledge support by the Department of Energy, Bridging Research Interactions through collaborative Development Grants in Energy (BRIDGE) program under contract DE-FOA-0000654-1588. Z.B. and L.S. acknowledge support by the National Science Foundation (DMR-1303178), and L.S. gratefully thanks the Kodak Graduate Fellowship.

\section{Notes and references}

1 P. J. Diemer, C. R. Lyle, Y. Mei, C. Sutton, M. M. Payne, J. E. Anthony, V. Coropceanu, J.-L. Brédas and O. D. Jurchescu, Adv. Mater., 2013, 25, 6956-6962.

2 S. S. Lee, C. S. Kim, E. D. Gomez, B. Purushothaman, M. F. Toney, C. Wang, A. Hexemer, J. E. Anthony and Y.-L. Loo, Adv. Mater., 2009, 21, 3605-3609.

3 D. H. Kim, J. T. Han, Y. D. Park, Y. Jang, J. H. Cho, M. Hwang and K. Cho, Adv. Mater., 2006, 18, 719-723.

4 D. H. Kim, D. Y. Lee, H. S. Lee, W. H. Lee, Y. H. Kim, J. I. Han and K. Cho, Adv. Mater., 2007, 19, 678-682.

5 S. Wang, M. Kappl, I. Liebewirth, M. Müller, K. Kirchhoff, W. Pisula and K. Müllen, Adv. Mater., 2012, 24, 417-420.
6 J. Chen, C. K. Tee, M. Shtein, D. C. Martin and J. Anthony, Org. Electron., 2009, 10, 696-703.

7 O. Goto, S. Tomiya, Y. Murakami, A. Shinozaki, A. Toda, J. Kasahara and D. Hobara, Adv. Mater., 2012, 24, 11171122.

8 C. Zhang, X. Zhang, X. Zhang, X. Fan, J. Jie, J. C. Chang, C.-S. Lee, W. Zhang and S.-T. Lee, Adv. Mater., 2008, 20, 1716-1720.

9 H. Li, B. C. K. Tee, J. J. Cha, Y. Cui, J. W. Chung, S. Y. Lee and Z. Bao, J. Am. Chem. Soc., 2012, 134, 2760-2765.

10 H. Li, B. C. K. Tee, G. Giri, J. W. Chung, S. Y. Lee and Z. Bao, Adv. Mater., 2012, 24, 2588-2591.

11 U. Takafumi, H. Yuri, U. Mayumi, T. Kazuo and T. Jun, Appl. Phys. Express, 2009, 2, 111501.

12 J. Soeda, Y. Hirose, M. Yamagishi, A. Nakao, T. Uemura, K. Nakayama, M. Uno, Y. Nakazawa, K. Takimiya and J. Takeya, Adv. Mater., 2011, 23, 3309-3314.

13 C. Mitsui, J. Soeda, K. Miwa, H. Tsuji, J. Takeya and E. Nakamura, J. Am. Chem. Soc., 2012, 134, 5448-5451.

14 L. L. Chua, P. K. H. Ho, H. Sirringhaus and R. H. Friend, Adv. Mater., 2004, 16, 1609-1615.

15 L. Qiu, J. A. Lim, X. Wang, W. H. Lee, M. Hwang and K. Cho, Adv. Mater., 2008, 20, 1141-1145.

16 C. Liu, Y. Li, M. V. Lee, A. Kumatani and K. Tsukagoshi, Phys. Chem. Chem. Phys., 2013, 15, 7917-7933.

17 Y. Yuan, G. Giri, A. L. Ayzner, A. P. Zoombelt, S. C. B. Mannsfeld, J. Chen, D. Nordlund, M. F. Toney, J. Huang and Z. Bao, Nat. Commun., 2014, 5, 3005, DOI: 10.1038/ncomms4005.

18 R. Z. Rogowski, A. Dzwilewski, M. Kemerink and A. A. Darhuber, J. Phys. Chem. C, 2011, 115, 11758-11762.

19 C.-Y. Liu and A. J. Bard, Chem. Mater., 2000, 12, 2353-2362.

20 A. Denneulin, A. Blayo, J. Bras and C. Neuman, Prog. Org. Coat., 2008, 63, 87-91.

21 C. E. Murphy, L. Yang, S. Ray, L. Yu, S. Knox and N. Stingelin, J. Appl. Phys., 2011, 110, 093523, DOI: 10.1063/1.3660779.

22 D. Khim, H. Han, K.-J. Baeg, J. Kim, S.-W. Kwak, D.-Y. Kim and Y.-Y. Noh, Adv. Mater., 2013, 25, 4302-4308.

23 Y.-H. Chang, S.-R. Tseng, C.-Y. Chen, H.-F. Meng, E.-C. Chen, S.-F. Horng and C.-S. Hsu, Org. Electron., 2009, 10, 741-746.

24 C.-Y. Chen, H.-W. Chang, Y.-F. Chang, B.-J. Chang, Y.-S. Lin, P.-S. Jian, H.-C. Yeh, H.-T. Chien, E.-C. Chen, Y.-C. Chao, H.-F. Meng, H.-W. Zan, H.-W. Lin, S.-F. Horng, Y.-J. Cheng, F.-W. Yen, I. F. Lin, H.-Y. Yang, K.-J. Huang and M.-R. Tseng, J. Appl. Phys., 2011, 110, 094501, DOI: 10.1063/1.3636398.

25 R. R. Søndergaard, M. Hösel and F. C. Krebs, J. Polym. Sci., Part B: Polym. Phys., 2013, 51, 16-34.

26 S.-R. Tseng, H.-F. Meng, K.-C. Lee and S.-F. Horng, Appl. Phys. Lett., 2008, 93, 153308, DOI: 10.1063/1.2999541.

27 R. Bollström, A. Määttänen, D. Tobjörk, P. Ihalainen, N. Kaihovirta, R. Österbacka, J. Peltonen and M. Toivakka, Org. Electron., 2009, 10, 1020-1023.

28 J. Soeda, T. Uemura, T. Okamoto, C. Mitsui, M. Yamagishi and J. Takeya, Appl. Phys. Express, 2013, 6, 076503. 
29 E. Cohen and E. J. Lightfoot, in Kirk-Othmer Encyclopedia of Chemical Technology, John Wiley \& Sons, Inc., 2000.

30 F. C. Krebs, J. Fyenbo and M. Jorgensen, J. Mater. Chem., 2010, 20, 8994-9001.

31 F. C. Krebs, Sol. Energy Mater. Sol. Cells, 2009, 93, 394-412.

32 A. Sandström, H. F. Dam, F. C. Krebs and L. Edman, Nat. Commun., 2012, 3, 1002.

33 H. A. Becerril, M. E. Roberts, Z. Liu, J. Locklin and Z. Bao, Adv. Mater., 2008, 20, 2588-2594.

34 Z. Liu, H. A. Becerril, M. E. Roberts, Y. Nishi and Z. Bao, IEEE Trans. Electron Devices, 2009, 56, 176-185.

35 G. Giri, E. Verploegen, S. C. B. Mannsfeld, S. AtahanEvrenk, D. H. Kim, S. Y. Lee, H. A. Becerril, A. AspuruGuzik, M. F. Toney and Z. Bao, Nature, 2011, 480, 504508.

36 V. Coropceanu, J. Cornil, D. A. da Silva Filho, Y. Olivier, R. Silbey and J.-L. Brédas, Chem. Rev., 2007, 107, 926952.

37 R. S. Sánchez-Carrera, S. Atahan, J. Schrier and A. n. AspuruGuzik, J. Phys. Chem. C, 2010, 114, 2334-2340.

38 J. Cornil, D. Beljonne, J. P. Calbert and J. L. Brédas, Adv. Mater., 2001, 13, 1053-1067.

39 Y. Diao, B. C. K. Tee, G. Giri, J. Xu, D. H. Kim, H. A. Becerril, R. M. Stoltenberg, T. H. Lee, G. Xue, S. C. B. Mannsfeld and Z. Bao, Nat. Mater., 2013, 12, 665-671.

40 R. D. Deegan, O. Bakajin, T. F. Dupont, G. Huber, S. R. Nagel and T. A. Witten, Nature, 1997, 389, 827-829.

41 S. S. Kim, S. I. Na, J. Jo, G. Tae and D. Y. Kim, Adv. Mater., 2007, 19, 4410-4415.

42 S.-S. Kim, S.-I. Na, S.-J. Kang and D.-Y. Kim, Sol. Energy Mater. Sol. Cells, 2010, 94, 171-175.

43 M. Ikawa, T. Yamada, H. Matsui, H. Minemawari, J. y. Tsutsumi, Y. Horii, M. Chikamatsu, R. Azumi, R. Kumai and T. Hasegawa, Nat. Commun., 2012, 3, 1176.

44 H. B. Akkerman, A. C. Chang, E. Verploegen, C. J. Bettinger, M. F. Toney and Z. Bao, Org. Electron., 2012, 13, 235-243.

45 M. Cavallini, P. Stoliar, J.-F. Moulin, M. Surin, P. Leclère, R. Lazzaroni, D. W. Breiby, J. W. Andreasen, M. M. Nielsen, P. Sonar, A. C. Grimsdale, K. Müllen and F. Biscarini, Nano Lett., 2005, 5, 2422-2425.

46 K. Nakayama, Y. Hirose, J. Soeda, M. Yoshizumi, T. Uemura, M. Uno, W. Li, M. J. Kang, M. Yamagishi, Y. Okada, E. Miyazaki, Y. Nakazawa, A. Nakao, K. Takimiya and J. Takeya, Adv. Mater., 2011, 23, 1626-1629.

47 P. S. Jo, A. Vailionis, Y. M. Park and A. Salleo, Adv. Mater., 2012, 24, 3269-3274.

48 Z. Zheng, K.-H. Yim, M. S. M. Saifullah, M. E. Welland, R. H. Friend, J.-S. Kim and W. T. S. Huck, Nano Lett., 2007, 7, 987-992.

49 M. Singh, H. M. Haverinen, P. Dhagat and G. E. Jabbour, Adv. Mater., 2010, 22, 673-685.

50 B. Derby, Annu. Rev. Mater. Res., 2010, 40, 395-414.

51 B.-J. de Gans and U. S. Schubert, Langmuir, 2004, 20, 77897793.

52 H. Sirringhaus, T. Kawase, R. H. Friend, T. Shimoda, M. Inbasekaran, W. Wu and E. P. Woo, Science, 2000, 290, 2123-2126.
53 H. Minemawari, T. Yamada, H. Matsui, J. y. Tsutsumi, S. Haas, R. Chiba, R. Kumai and T. Hasegawa, Nature, 2011, 475, 364-367.

54 A. C. Arias, S. E. Ready, R. Lujan, W. S. Wong, K. E. Paul, A. Salleo, M. L. Chabinyc, R. Apte, R. A. Street, Y. Wu, P. Liu and B. Ong, Appl. Phys. Lett., 2004, 85, 3304-3306.

55 A. Abdellah, B. Fabel, P. Lugli and G. Scarpa, Org. Electron., 2010, 11, 1031-1038.

56 D. Vak, S.-S. Kim, J. Jo, S.-H. Oh, S.-I. Na, J. Kim and D.-Y. Kim, Applied Physics Letters, 2007, 91, 081102, DOI: 10.1063/1.2772766.

57 S. F. Tedde, J. Kern, T. Sterzl, J. Fürst, P. Lugli and O. Hayden, Nano Lett., 2009, 9, 980-983.

58 L.-M. Chen, Z. Hong, W. L. Kwan, C.-H. Lu, Y.-F. Lai, B. Lei, C.-P. Liu and Y. Yang, ACS Nano, 2010, 4, 4744-4752.

59 T. Ishikawa, M. Nakamura, K. Fujita and T. Tsutsui, Appl. Phys. Lett., 2004, 84, 2424-2426.

60 Y. Aoki, M. Shakutsui and K. Fujita, Thin Solid Films, 2009, 518, 493-496.

61 K. Fujita, T. Ishikawa and T. Tsutsui, Jpn. J. Appl. Phys., Part 1, 2002, 41, L70.

62 M. Shao, S. Das, K. Xiao, J. Chen, J. K. Keum, I. N. Ivanov, G. Gu, W. Durant, D. Li and D. B. Geohegan, J. Mater. Chem. C, 2013, 1, 4384-4390.

63 R. C. Tenent, T. M. Barnes, J. D. Bergeson, A. J. Ferguson, B. To, L. M. Gedvilas, M. J. Heben and J. L. Blackburn, Adv. Mater., 2009, 21, 3210-3216.

64 K. X. Steirer, M. O. Reese, B. L. Rupert, N. Kopidakis, D. C. Olson, R. T. Collins and D. S. Ginley, Sol. Energy Mater. Sol. Cells, 2009, 93, 447-453.

$65 \mathrm{~J} . \mathrm{Ju}$, Y. Yamagata and T. Higuchi, Adv. Mater., 2009, 21, 4343-4347.

66 J. A. Lim, H. S. Lee, W. H. Lee and K. Cho, Adv. Funct. Mater., 2009, 19, 1515-1525.

67 A. A. Virkar, S. Mannsfeld, Z. Bao and N. Stingelin, Adv. Mater., 2010, 22, 3857-3875.

68 S. Liu, W. M. Wang, A. L. Briseno, S. C. E. Mannsfeld and Z. Bao, Adv. Mater., 2009, 21, 1217-1232.

69 S. S. Lee and Y.-L. Loo, Annu. Rev. Chem. Biomol. Eng., 2010, 1, 59-78.

70 A. M. Hiszpanski and Y.-L. Loo, Energy Environ. Sci., 2014, 7, 592-608.

71 G. Giri, E. Verploegen, S. C. B. Mannsfeld, S. AtahanEvrenk, D. H. Kim, S. Y. Lee, H. A. Becerril, A. AspuruGuzik, M. F. Toney and Z. A. Bao, Nature, 2011, 480, 504-U124.

72 Y. Diao, B. C. K. Tee, G. Giri, J. Xu, D. H. Kim, H. A. Becerril, R. M. Stoltenberg, T. H. Lee, G. Xue, S. C. B. Mannsfeld and Z. N. Bao, Nat. Mater., 2013, 12, 665-671.

73 B. D. Naab, S. Himmelberger, Y. Diao, K. Vandewal, P. Wei, B. Lussem, A. Salleo and Z. Bao, Adv. Mater., 2013, 25, 46634667.

74 J. A. Lim, W. H. Lee, H. S. Lee, J. H. Lee, Y. D. Park and K. Cho, Adv. Funct. Mater., 2008, 18, 229-234.

75 N. D. Treat, J. A. N. Malik, O. Reid, L. Yu, C. G. Shuttle, G. Rumbles, C. J. Hawker, M. L. Chabinyc, P. Smith and N. Stingelin, Nat. Mater., 2013, 12, 628-633. 
76 H. Li, B. C. K. Tee, J. J. Cha, Y. Cui, J. W. Chung, S. Y. Lee and Z. Bao, J. Am. Chem. Soc., 2012, 134, 2760-2765.

77 Y. Diao, A. S. Myerson, T. A. Hatton and B. L. Trout, Langmuir, 2011, 27, 5324-5334.

78 Y. Diao, M. E. Helgeson, Z. A. Siam, P. S. Doyle, A. S. Myerson, T. A. Hatton and B. L. Trout, Cryst. Growth Des., 2011, 12, 508-517.

79 S. S. Lee, S. Muralidharan, A. R. Woll, M. A. Loth, Z. Li, J. E. Anthony, M. Haataja and Y.-L. Loo, Chem. Mater., 2012, 24, 2920-2928.

80 Y. Ito, A. A. Virkar, S. Mannsfeld, J. H. Oh, M. Toney, J. Locklin and Z. Bao, J. Am. Chem. Soc., 2009, 131, 93969404.

81 J. Rivnay, L. H. Jimison, J. E. Northrup, M. F. Toney, R. Noriega, S. Lu, T. J. Marks, A. Facchetti and A. Salleo, Nat. Mater., 2009, 8, 952-958.

82 A. L. Briseno, S. C. B. Mannsfeld, M. M. Ling, S. Liu, R. J. Tseng, C. Reese, M. E. Roberts, Y. Yang, F. Wudl and Z. Bao, Nature, 2006, 444, 913-917.

83 S. Liu, A. L. Briseno, S. C. B. Mannsfeld, W. You, J. Locklin, H. W. Lee, Y. Xia and Z. Bao, Adv. Funct. Mater., 2007, 17, 2891-2896.

84 A. Hara and N. Sasaki, J. Appl. Phys., 2000, 88, 33493353.

85 L. Li, P. Gao, K. C. Schuermann, S. Ostendorp, W. Wang, C. Du, Y. Lei, H. Fuchs, L. De Cola, K. Muellen and L. Chi, J. Am. Chem. Soc., 2010, 132, 8807-8809.

86 S. Wo, R. L. Headrick and J. E. Anthony, J. Appl. Phys., 2012, 111, 073716, DOI: 10.1063/1.3698203.

87 D. J. Mascaro, M. E. Thompson, H. I. Smith and V. Bulović, Org. Electron., 2005, 6, 211-220.

88 K. C. Dickey, J. E. Anthony and Y. L. Loo, Adv. Mater., 2006, 18, 1721-1726.

89 S. S. Lee, S. B. Tang, D.-M. Smilgies, A. R. Woll, M. A. Loth, J. M. Mativetsky, J. E. Anthony and Y.-L. Loo, Adv. Mater., 2012, 24, 2692-2698.

90 C. Liu, T. Minari, X. Lu, A. Kumatani, K. Takimiya and K. Tsukagoshi, Adv. Mater., 2011, 23, 523-526.

91 H. Ullah Khan, R. Li, Y. Ren, L. Chen, M. M. Payne, U. S. Bhansali, D.-M. Smilgies, J. E. Anthony and A. Amassian, ACS Appl. Mater. Interfaces, 2013, 5, 23252330.

92 D. J. Gundlach, J. E. Royer, S. K. Park, S. Subramanian, O. D. Jurchescu, B. H. Hamadani, A. J. Moad, R. J. Kline, L. C. Teague, O. Kirillov, C. A. Richter, J. G. Kushmerick, L. J. Richter, S. R. Parkin, T. N. Jackson and J. E. Anthony, Nat. Mater., 2008, 7, 216-221.

93 L. H. Jimison, M. F. Toney, I. McCulloch, M. Heeney and A. Salleo, Adv. Mater., 2009, 21, 1568-1572.

94 M. Brinkmann and J. C. Wittmann, Adv. Mater., 2006, 18, 860-863.

95 C. De Rosa, C. Park, E. L. Thomas and B. Lotz, Nature, 2000, 405, 433-437.

96 A. R. Aiyar, J.-I. Hong, R. Nambiar, D. M. Collard and E. Reichmanis, Adv. Funct. Mater., 2011, 21, 2652-2659.

97 T. Komino, H. Kuwabara, M. Ikeda, M. Yahiro, K. Takimiya and C. Adachi, Langmuir, 2013, 29, 9592-9597.
98 P. J. Diemer, C. R. Lyle, Y. Mei, C. Sutton, M. M. Payne, J. E. Anthony, V. Coropceanu, J.-L. Brédas and O. D. Jurchescu, Adv. Mater., 2013, 25, 6956-6962.

99 W. Pisula, A. Menon, M. Stepputat, I. Lieberwirth, U. Kolb, A. Tracz, H. Sirringhaus, T. Pakula and K. Mullen, Adv. Mater., 2005, 17, 684-689.

100 R. L. Headrick, S. Wo, F. Sansoz and J. E. Anthony, Appl. Phys. Lett., 2008, 92, 063302, DOI: 10.1063/1.2839394.

101 J. Chang, C. Chi, J. Zhang and J. Wu, Adv. Mater., 2013, 25, 6442-6447.

102 V. Podzorov, E. Menard, A. Borissov, V. Kiryukhin, J. A. Rogers and M. E. Gershenson, Phys. Rev. Lett., 2004, 93, 086602, DOI: 10.1103/PhysRevLett.93.086602.

103 S. C. B. Mannsfeld, J. Locklin, C. Reese, M. E. Roberts, A. J. Lovinger and Z. Bao, Adv. Funct. Mater., 2007, 17, 1617-1622.

104 J. L. Bredas, J. P. Calbert, D. A. da Silva and J. Cornil, Proc. Natl. Acad. Sci. U. S. A., 2002, 99, 5804-5809.

105 V. Coropceanu, J. Cornil, D. A. da Silva, Y. Olivier, R. Silbey and J. L. Bredas, Chem. Rev., 2007, 107, 926-952.

106 C. W. Sele, B. K. C. Kjellander, B. Niesen, M. J. Thornton, J. B. P. H. van der Putten, K. Myny, H. J. Wondergem, A. Moser, R. Resel, A. J. J. M. van Breemen, N. van Aerle, P. Heremans, J. E. Anthony and G. H. Gelinck, Adv. Mater., 2009, 21, 4926-4931.

107 M. Le Berre, Y. Chen and D. Baigl, Langmuir, 2009, 25, 2554-2557.

108 R. Z. Rogowski and A. A. Darhuber, Langmuir, 2010, 26, 11485-11493.

109 T. Uemura, Y. Hirose, M. Uno, K. Takimiya and J. Takeya, Appl. Phys. Express, 2009, 2, 111501, DOI: 10.1143/ apex.2.111501.

110 W. H. Lee, D. H. Kim, Y. Jang, J. H. Cho, M. Hwang, Y. D. Park, Y. H. Kim, J. I. Han and K. Cho, Appl. Phys. Lett., 2007, 90, 132106, DOI: 10.1063/1.2717087.

111 T. Lei and J. Pei, J. Mater. Chem., 2012, 22, 785-798.

112 N. Liu, Y. Zhou, N. Ai, C. Luo, J. Peng, J. Wang, J. Pei and Y. Cao, Langmuir, 2011, 27, 14710-14715.

113 W. Jiang, Y. Zhou, H. Geng, S. Jiang, S. Yan, W. Hu, Z. Wang, Z. Shuai and J. Pei, J. Am. Chem. Soc., 2010, 133, 1-3.

114 D. H. Kim, D. Y. Lee, H. S. Lee, W. H. Lee, Y. H. Kim, J. I. Han and K. Cho, Adv. Mater., 2007, 19, 678-682.

115 J. H. Oh, H. W. Lee, S. Mannsfeld, R. M. Stoltenberg, E. Jung, Y. W. Jin, J. M. Kim, J.-B. Yoo and Z. Bao, Proc. Natl. Acad. Sci. U. S. A., 2009, 106, 6065-6070.

116 G. H. Yu, A. Y. Cao and C. M. Lieber, Nat. Nanotechnol., 2007, 2, 372-377.

117 Y. Z. Long, M. Yu, B. Sun, C. Z. Gu and Z. Y. Fan, Chem. Soc. Rev., 2012, 41, 4560-4580.

118 C. M. Schroeder, E. S. G. Shaqfeh and S. Chu, Macromolecules, 2004, 37, 9242-9256.

119 A. B. Marciel, M. Tanyeri, B. D. Wall, J. D. Tovar, C. M. Schroeder and W. L. Wilson, Adv. Mater., 2013, 25, 6398-6404.

120 W. H. Lee, J. H. Cho and K. Cho, J. Mater. Chem., 2010, 20, 2549-2561. 